\title{
Modulatory effects of happy mood on performance monitoring: Insights from error-related brain potentials
}

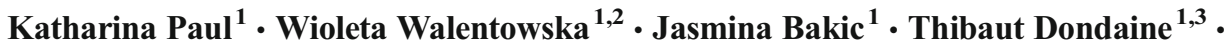 \\ Gilles Pourtois ${ }^{1}$
}

Published online: 17 September 2016

(C) Psychonomic Society, Inc. 2016

\begin{abstract}
Goal-adaptive behavior requires the rapid detection of conflicts between actions and intentions or goals. Although many studies have focused in the past on the influence of negative affect on this cognitive control process (and more specifically, on error monitoring), little is known about the possible modulatory effects of positive affect on it. To address this question, we used a standard (positive) mood induction procedure (based on guided imagery) and asked participants to carry out a speeded go/no-go task while high-density electroencephalography was recorded concurrently. As a control condition, we used a group with neutral mood. Event-related potential results showed that the error-related negativity (ERN) component, reflecting early error detection within the dorsal anterior cingulate cortex, was not influenced by happy mood. In contrast, the subsequent error positivity (Pe) component, related to the appraisal of the motivational significance of errors, was reliably smaller in the happy than in the neutral mood group. Complementing source localization analyses showed that this effect was explained by decreased activation within the posterior cingulate and insular cortices. These results were obtained in the absence of group differences
\end{abstract}

Electronic supplementary material The online version of this article (doi:10.3758/s13415-016-0466-8) contains supplementary material, which is available to authorized users.

Katharina Paul

Katharina.Paul@Ugent.be

1 Department of Experimental Clinical \& Health Psychology, Ghent University, Henri Dunantlaan 2, 9000 Gent, Belgium

2 Psychophysiology Laboratory, Institute of Psychology, Jagiellonian University, Krakow, Poland

3 Behavior and Basal Ganglia Laboratory (EA 4712), University of Rennes 1, Rennes, France regarding behavioral performance and tonic arousal. These findings suggest that happy mood likely decreases and changes the motivational significance of worse-than-expected events $(\mathrm{Pe})$, while leaving their earlier automatic detection (ERN) unaltered. We discuss these new results in terms of dynamic changes in the complex interplay of performance monitoring with motivation.

Keywords ERP $\cdot$ Mood $\cdot$ Motivation $\cdot$ Cognitive control

Human behavior is characterized by a high amount of flexibility, which is necessary to deal efficiently with rapidly changing demands in the environment. This ability stems from dedicated cognitive control mechanisms that monitor the occurrence of deviance between intended and actual actions, and if such deviance is detected, in turn trigger specific remedial processes (Botvinick \& Braver, 2015). In this framework, performance monitoring (PM) is usually achieved by the processing of external incentives (such as positive or negative feedback) or internal/motor cues (such as correct responses or response errors; Ullsperger, Fischer, Nigbur, \& Endrass, 2014). Feedback-locked and response-locked PM is thought to operate via dopaminergic-dependent reward prediction error mechanisms or signals that influence specific fronto-striatal loops in the human brain (Frank, Woroch, \& Curran, 2005; Holroyd \& Coles, 2002; Walsh \& Anderson, 2012). Interestingly, accumulating evidence is showing that $\mathrm{PM}$ is not immune to changes in the affective state of the participant or in specific motivational drives (Koban \& Pourtois, 2014; Olvet \& Hajcak, 2008; Weinberg, Riesel, \& Hajcak, 2012). More specifically, response-locked PM brain mechanisms appear to be reliably influenced by trait negative affect (such as anxiety/apprehension/worry; Moser, Moran, Schroder, Donnellan, \& Yeung, 2013; Olvet \& Hajcak, 
2008; Pizzagalli, 2014), as well as by induced negative emotion (Wiswede, Münte, Goschke, \& Rüsseler, 2009; Wiswede, Münte, \& Rüsseler, 2009). By comparison, much less is known about potential modulation of PM by emotions of positive valence. This paucity is somewhat surprising at first sight, given that positive emotions fuel resilience and wellbeing (Sheldon \& King, 2001), and they are usually assigned a special, protective or beneficial, role in core cognitive processes, such as attention, reasoning, or creativity (Fredrickson, 2001). Accordingly, in this study we set out to test the prediction that positive emotions could perhaps influence PM, with a focus on early response-locked error-monitoring processes, which had previously been found to be susceptible to effects associated with negative emotions.

At the electroencephalographic (EEG) level, error monitoring provides a very good insight into PM processes and their malleability by affect or motivation, given that this process is captured by systematic amplitude variations of two welldocumented event-related potentials (ERPs): the errorrelated negativity (ERN or $\mathrm{Ne}$ ) and the error positivity (Pe) (Falkenstein, Hohnsbein, Hoormann, \& Blanke, 1991; Gehring, Goss, Coles, Meyer, \& Donchin, 1993; Ullsperger, Danielmeier, \& Jocham, 2014). Whereas the ERN component reflects the early, perhaps automatic, detection of a discrepancy (in terms of motor representations) between the incorrect executed and the correct desired or intended action (Coles, Scheffers, \& Holroyd, 2001; Gehring et al., 1993), the subsequent $\mathrm{Pe}$ is usually related to the conscious appraisal of response errors and/or to the processing of their motivational significance (Falkenstein, Hoormann, Christ, \& Hohnsbein, 2000; Koban \& Pourtois, 2014; Nieuwenhuis, Ridderinkhof, Blom, Band, \& Kok, 2001; Ridderinkhof, Ramautar, \& Wijnen, 2009). Hence, the ERN and Pe likely reflect two distinctive processes during error monitoring and PM more broadly defined.

Despite its ultra-fast neurophysiological time course (as it is usually elicited $0-100 \mathrm{~ms}$ after error commission over fronto-central electrodes along the midline), and high degree of automaticity, the amplitude of the ERN varies however with motivational factors (e.g., when accuracy is emphasized; see Gehring et al., 1993) or emotional variables (such as trait anxiety; see Aarts \& Pourtois, 2010) suggesting that it is not only reflecting motor cognition per se, but probably already capturing emotional appraisal processes during PM (for a review, see Olvet \& Hajcak, 2008). For example, the ERN amplitude is usually increased for internalizing traits or disorders, including depression (Chiu \& Deldin, 2007; A. J. Holmes \& Pizzagalli, 2008), anxiety (Aarts \& Pourtois, 2010; Hajcak, McDonald, \& Simons, 2003a), and obsessive compulsive disorder (Endrass \& Ullsperger, 2014). By comparison, its amplitude is usually decreased in externalizing traits or disorders, such as in participants with cocaine dependence (Franken, van Strien, Franzek, \& van de Wetering, 2007) or impulsive personality characteristics (Ruchsow, Spitzer, Grön, Grothe, \& Kiefer, 2005). Growing evidence showing a reliable increase of the ERN amplitude with negative affect at a nonclinical level (Hajcak, McDonald, \& Simons, 2004; Luu, Collins, \& Tucker, 2000; Vaidyanathan, Nelson, \& Patrick, 2012) has been extended by studies examining manipulated sadness, short-term negative affect, and induced helplessness (Olvet \& Hajcak, 2012; Pfabigan et al., 2013; Wiswede, Münte, Goschke, \& Rüsseler, 2009; Wiswede, Münte, \& Rüsseler, 2009). All in all, these studies concur in suggesting that negative affect (conceived as either a trait or a state) reliably increases the ERN component.

By comparison, the subsequent Pe component, peaking 145-300 ms after error commission over more posterior central areas than the ERN/Ne, considered to covary closely with the degree of error awareness or the amount of salience induced by response errors (Overbeek, Nieuwenhuis, \& Ridderinkhof, 2005), appears to be much less systematically influenced by negative affect. However, scattered evidence has suggested that an overactive ERN usually goes along with a decreased $\mathrm{Pe}$, as has been demonstrated in participants reporting high levels of trait negative affect (Hajcak et al., 2004), in those with clinical depression (Aarts, Vanderhasselt, Otte, Baeken, \& Pourtois, 2013; Chiu \& Deldin, 2007; A. J. Holmes \& Pizzagalli, 2010; Olvet, Klein, \& Hajcak, 2010; Schrijvers et al., 2009; Schroder, Moran, Infantolino, \& Moser, 2013), or in studies inducing threat as a negative emotional state (Moser, Hajcak, \& Simons, 2005). The lack of a clear understanding of the effects of (negative) affect on the Pe is also reinforced by the fact that in many studies the authors usually focus on the ERN exclusively, and do not report possible effects on the subsequent Pe component.

Although still debated in the literature, the enhanced ERN amplitudes accompanying negative affect most likely reflect the higher significance of response errors for these participants - that is, they recruit more cognitive resources to detect errors - whereas the reduced Pe amplitude could reflect a lower awareness or salience of error commission, even though it appears difficult to reconcile these two opposing accounts. At any rate, an overactive ERN in negative affect is in line with both the assumption of a mood congruency effect during PM (Rusting, 1998), as well as with the divergent functional significance of specific mood states (Fredrickson, 2001, 2004). In the latter framework, mood does not simply trigger changes in the approach-versus-avoidance motivational system in a way that is compatible with the actual mood content (negative mood yielding avoidance, and positive mood fostering approach). Instead, distinct mood states are characterized by different functions that can, in turn, influence cognition and behavior in nontransparent ways. According to this model, negative mood signals a potentially threatening environment, whereby the individual puts more efforts toward timely detection and eventual avoidance of possible dangers or threats. 
Presumably, unwanted response errors are aversive and belong to this category, and their swift detection at the ERN level may therefore be gated with the encounter or experience of negative affect. In contrast, positive mood signals a safe environment, where a more creative and heuristic processing style is usually promoted, leading to a broadening of attention and the building of (additional) mental resources (Fredrickson, 1998, 2001). Therefore, in a happy mood state, there is no need for increased error monitoring. Furthermore, this state likely shields the individual from experiencing negative affect or distress when encountering worse-than-expected events, and as such, it helps maintain the current and pleasurable mood state (Schwarz \& Bless, 1991). However, whether or not the latter affective state (positive mood) leads to a change in the amplitude of the ERN or the Pe remains unsettled. As a matter of fact, discrepant findings have been reported in the past regarding the modulatory effects of positive affect on early error-monitoring processes at the ERN level, whereas results for Pe amplitudes have usually not been scrutinized or reported. Some studies have reported that smaller ERN amplitudes were related to higher life satisfaction (Larson, Good, \& Fair, 2010) or religiosity, which is linked to a general positive view on life (Inzlicht, McGregor, Hirsh, \& Nash, 2009), or to positive affect after watching movie clips (van Wouwe, Band, \& Ridderinkhof, 2011). In another study, the opposite pattern was found: Bakic, Jepma, De Raedt, and Pourtois (2014) found a larger ERN amplitude during a probabilisticlearning task after positive mood induction with guided imagery. Moreover, other studies have actually failed to find a reliable influence of positive emotions on the size of the ERN component (e.g., Luu et al., 2000, who assessed positive affect using the PANAS, or Wiswede, Münte, Goschke, and Rüsseler (2009) who placed pleasant IAPS pictures before responses). This discrepancy likely stems from the fact that different methods to induce and measure positive emotions or mood (in a very broad sense) were used across these different studies. To measure mood, verbal self-reports or subjective ratings are often applied, but they show specific limitations, such as introspection, unlike more objective psychophysiological measurements. To induce a positive emotional state, very often automatic emotional reactions are provoked using specific emotional material (pictures, music, films) or rewards/punishments (for reviews, see Gilet, 2008; Westermann et al., 1996). However, because the same material is used for all participants (to seek standardization), it lacks individualization and may therefore be suboptimal. For this reason, more recently induction techniques based on the use of guided imagery and the recall of personal autobiographical information have been proposed as alternatives to overcome this limitation and eventually to induce more potent participantspecific mood states with enhanced ecological validity
(Bakic et al., 2014; E. A. Holmes, Mathews, Dalgleish, \& Mackintosh, 2006; E. A. Holmes, Oughtrey, \& Connor, 2008; Kross, Davidson, Weber, \& Ochsner, 2009; Vanlessen, De Raedt, Mueller, Rossi, \& Pourtois, 2015; Vanlessen, Rossi, De Raedt, \& Pourtois, 2013, 2014).

The goal of this study was to gain insight into possible modulatory effects of positive mood (once it is induced and maintained) on error monitoring, with a focus on the ERN and Pe ERP components. In light of this aim, we directly manipulated the current mood state of the participants by means of guided imagery (E. A. Holmes et al., 2006; E. A. Holmes et al., 2008) while they performed a standard speeded go/no-go task/procedure. This task is suited to produce a large number of unwanted response errors and has been previously validated in a number of studies (Aarts, De Houwer, \& Pourtois, 2012; Aarts \& Pourtois, 2010; Aarts et al., 2013; Vocat, Pourtois, \& Vuilleumier, 2008). This mood induction procedure (MIP) has been validated in our laboratory across different studies (see Bakic et al., 2014; Vanlessen et al., 2013, 2014). Using it, we induced either a happy or a neutral mood in a between-subjects design. Once a mood had been induced, participants carried out a speeded go/nogo task while 64-channel EEG was recorded concurrently, to study the neurophysiology of error monitoring (ERN and Pe components) carefully. The "broaden-and-build" theory of positive emotions (Fredrickson, 2004) provides an important framework from which some predictions could be derived in the present case. In this framework, positive mood is thought to increase creativity (Isen, 2008; Subramaniam, Kounios, Parrish, \& Jung-Beeman, 2009) and cognitive flexibility (Nadler, Rabi, \& Minda, 2010), and to broaden attention (Vanlessen et al., 2013, 2014), while it can also impair specific components of executive function, including planning, task-switching, and inhibition abilities (Mitchell \& Phillips, 2007), because of the enhanced distractibility accompanying this specific mood state (Dreisbach \& Goschke, 2004). Hence, in light of this evidence, positive mood could very well interfere with, rather than increase, performance (accuracy, speed), early error-monitoring processes, and behavioral adaptation following error commission (i.e., posterror slowing). This might eventually be translated into a blunted ERN or Pe component during the rapid monitoring of response errors in individuals experiencing a happy mood, as compared to an active control condition with neutral mood content. Besides the changes in the current mood state captured by subjective ratings, we also measured physiological arousal concurrently to assess whether or not any modulatory effects of positive mood on error monitoring would be related to changes in the autonomic nervous system. 


\section{Method}

\section{Participants}

Fifty undergraduate students from Ghent University took part in the study in exchange for $€ 30$ compensation. All of them were right-handed, reported normal or corrected-to-normal vision, and had no history of psychiatric or neurological disorders. The study was approved by the local ethics committee, and all participants gave written informed consent prior to participation. Participants were randomly allocated to either a positive or a neutral mood condition ( $n=25$ per group). The data of three participants were excluded due to failures of mood induction (see Bakic, De Raedt, Jepma, \& Pourtois, 2015; Bakic et al., 2014): Within the neutral mood condition, two participants were excluded because their level of happiness increased and stayed on a very high level after the MIP, as compared to the rest of the neutral group (more than $1.8 \mathrm{SDs}$ above the mean), and within the happy mood group, the data of one participant had to be excluded due to a decrease in levels of happiness following the mood induction, relative to the baseline measurement prior to it (1.8 SDs below the mean). Furthermore, three participants (two from the happy group) had to be excluded due to technical problems during EEG data acquisition. Hence, 22 participants per mood group were eventually included in the final sample. These two groups were matched for gender and age (happy group $M_{\text {age }}$ $=21.8$ years, $S D=2.52,14$ females, eight males; neutral group $M_{\text {age }}=22.4$ years, $S D=2.26,15$ females, seven males).

\section{Mood induction}

A previously validated MIP was used (see Bakic et al., 2014; Vanlessen et al., 2013, 2014). In a between-subjects design, either positive or neutral mood was induced by means of an imagery procedure in which participants were instructed to vividly imagine and reexperience a specific autobiographical memory episode (E. A. Holmes et al., 2006; E. A. Holmes et al., 2008). Participants were kept naive regarding the purpose of the procedure, which they were told concerned episodic memory abilities (and not about emotional reexperiencing). Prior to the MIP, participants were trained in multisensory imaging from their own perspective with a standard four-step imagery exercise (manipulating a lemon; E. A. Holmes et al., 2006; E. A. Holmes et al., 2008). Then they had to choose an appropriate episodic memory that had happened at least one week before and that either made them feel very happy (positive mood group) or did not elicit any specific emotions but was linked to a physical activity (neutral mood group). We chose this specific instruction in the neutral mood group so as to try to balance levels of arousal (after the MIP) with the happy mood group (in which both valence and arousal usually increase; see Bakic et al., 2015). Table 1 provides a summary of the main
Table 1 Content of the reported memories

\begin{tabular}{llll}
\hline Neutral Memories & \multicolumn{3}{c}{ Positive Memories } \\
\hline House \& garden work & $(6)$ & Activity with friends & $(6)$ \\
Running, jogging & $(5)$ & Sports (excitement) & $(5)$ \\
Prepare food & $(5)$ & Vacation, relaxation & $(5)$ \\
Swimming & $(3)$ & Mating behavior & $(4)$ \\
Walking, hiking & $(3)$ & Buy wanted thing & $(3)$ \\
Riding bike & $(2)$ & Family & $(2)$ \\
Moving flat & $(1)$ & Childhood memory & $(1)$ \\
\hline
\end{tabular}

The participants in the neutral mood group focused mostly on sportsrelated activities (in line with the instructions), whereas those in the positive mood group primarily retrieved activities that included a social component.

memory contents retrieved by the participants, which shows that in the neutral group they mainly recalled sports-related activities, whereas in the positive mood group they primarily used activities characterized by the presence of a social component. Next, the participants closed their eyes and tried to imagine the situation as vividly as possible two times for $60 \mathrm{~s}$, interrupted by precise questions asked by the experimenter about the sensations and details, to encourage concrete imaginations (E. A. Holmes et al., 2008; Watkins \& Moberly, 2009) and ensure that regardless of the mood condition, participants engaged similarly in vivid mental imagery. Finally, participants were asked (on the basis of a rating scale with five points, ranging from not at all to completely) how well they could imagine the situation from their own perspective; this was used as a short manipulation check to assess how strongly they could reexperience the desired memories (in their mind's eyes). The MIP was repeated after each block of the go/no-go task (every 5 min, three times), with the aim of maintaining the targeted mood state throughout the whole experimental session.

To check (at the subjective level) the current mood state before the first and after every MIP, participants were asked to mark on a $10-\mathrm{cm}$ horizontal visual analogue scale their current feeling of happiness, pleasantness, and sadness. The left anchor was labeled with neutral and the right one with as happy/ pleasant/sad as I can imagine. Furthermore, participants had to rate their current arousal level with a self-assessment manikin for arousal (Bradley \& Lang, 1994).

\section{Task}

Participants performed a modified version of a speeded go/nogo task that had previously been used and validated in different studies (Aarts et al., 2013; Pourtois, 2011; Vocat et al., 2008); see Fig. 1 for an overview. The visual stimuli consisted of a square or a diamond presented in the center of a white screen. Each trial started with a fixation cross $(500 \mathrm{~ms})$, then a black square or diamond was presented for a variable time 
A

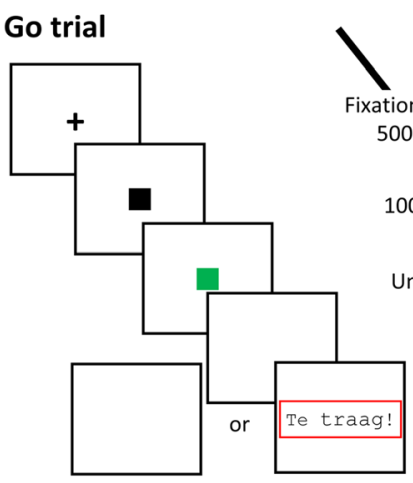

Fig. 1 Stimuli and task. a On each trial, a black square was first presented. After a variable interval (randomly varying between 1,000 and 2,000 ms), this black square (two-thirds, go trials) became green and kept its initial orientation (either square or diamond). b On the

interval (between 1,000 and 2,000 ms, to keep uncertainty of the target time high). Then this geometric figure became either green or orange, while its in-plane orientation either remained identical (square-square or diamond-diamond sequence) or was swapped (square-diamond or diamond-square sequence). This visual stimulus remained on the screen for $1,000 \mathrm{~ms}$ or until a buttonpress. Participants had to perform a speeded color-plus-shape discrimination task, in which both speed and accuracy were emphasized. When the geometric figure turned green and kept its original shape (two-thirds of the trials; go trials), participants had to press a predefined key on the response box as fast as possible with their right index finger. If the geometric figures turned orange (one-sixth of the trials) or changed shape (one-sixth of the trials; all corresponding to no-go trials), participants then had to withhold responding. To ensure that every participant would commit a sufficient number of response errors (i.e., false alarms on nogo trials) without creating excessive frustration or blurring the task rules, we used a strict reaction time (RT) cutoff (see also Aarts et al., 2012; Aarts \& Pourtois, 2010; Vocat et al., 2008). On each and every (go) trial, the RT was compared against an arbitrary cutoff. If the RT speed was above this limit (slow hit trial, $\mathrm{SH}$ ), then negative feedback was provided $1,000 \mathrm{~ms}$ after response onset ("too slow," written in Dutch, was presented for $500 \mathrm{~ms}$ in the center of the screen). No feedback was provided after so-called fast hits (FHs; i.e., the RT speed was below the cutoff) or errors, to increase internal monitoring in these cases. Unknown to the participants, this cutoff was calculated during specific calibration blocks that preceded the experimental blocks. During the first three experimental blocks, participants had to be $10 \%$ faster than the mean calculated during the (yoked) calibration blocks, and 20\% faster during the last (fourth) experimental block. The added value of this procedure is that the RT cutoff is calculated for each participant separately and adjusted during the experimental
B

\section{NoGo trial}

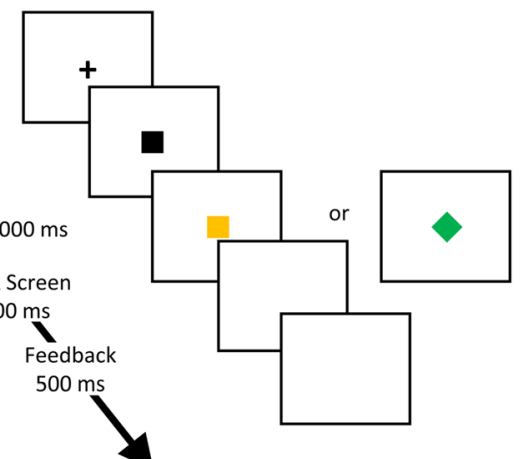

remaining trials (one-third), it became either orange or green with a change in orientation (no-go trials). Go and no-go trials were shown in a random order

session to deal with the inherent interindividual variability in RT speed, as well as the unspecific effects of time and habituation/learning (intra-individual variability; see Vocat et al., 2008). The experiment consisted of a practice block of 12 trials (four go trials, and four no-go trials of each type), two calibration blocks of 14 trials (ten go and two no-go trials of each type), and four experimental blocks of 84 trials each. Each calibration block was followed by two test blocks. Trial presentation was randomized within blocks. Stimuli were shown on a 21-in. CRT screen, and the task was programmed and executed using E-Prime (Version 2.0; Psychology Software Tools Inc., Sharpsburg, PA).

\section{Recording and preprocessing of electrophysiological data}

EEG was recorded using a 64-channel Biosemi Active Two system (www.biosemi.com). EEG was sampled at $512 \mathrm{~Hz}$ and referenced to the common mode sense (CMS) active electrode-driven right leg passive electrodes. The EEG was preprocessed offline with Brain Vision Analyzer 2.0, using a standard scheme of data transformation meant to extract response-locked ERPs (Keil et al., 2014). First, a 0.016-Hz high-pass filter was applied, and the data were re-referenced using the common average of all electrodes. ${ }^{1}$ Individual epochs were segmented using a \pm 500 -ms interval around the response onset. Eye blinks were removed automatically with an ocular correction for blinks (Gratton, Coles, \& Donchin, 1983), by using the difference amplitude of the two electrodes attached above and below the left eye, respectively. Each epoch was baseline corrected using a 200-ms time interval (-500

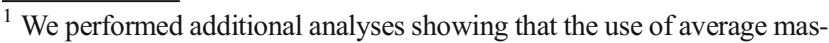
toids, as opposed to the common average reference (see Supplementary Fig. 1), did not change the main outcome of the study (i.e., happy mood influences the Pe component selectively).
} 
to $-300 \mathrm{~ms}$ prior to the response). Artifact rejection was based on a $\pm 70-\mu \mathrm{V}$ amplitude cutoff. Using this criterion, at least 70 $\%$ of the individual segments were kept and included in the averages, with no significant group difference in the amplitude cutoffs $\left(M_{\mathrm{Pos}}=72.6, S D=9.29, M_{\mathrm{Neu}}=75.2, S D=9.82\right)[t(1$, $42)=0.915, p=.37, d=0.27]$. Individual trials were averaged separately for each condition, and finally, a 30-Hz low-pass filter was applied before grand-average response-locked ERP waveforms were computed.

Electrodermal activity (EDA) was recorded continuously (512-Hz sampling rate, using the same parameters as for the EEG recording) via two bipolar electrodes that were attached to the volar surfaces of the distal phalanges of the left index and middle fingers (i.e., of the nondominant hand). Participants were instructed to comfortably lay their left forearm on the table and were asked not to move it during the experimental blocks.

\section{Data analysis}

\section{Analysis of mood manipulation effects and behavioral da-} ta For these and all subsequent analyses, the significance alpha cutoff was set to .05 . To check for the efficiency of the MIP, we used a mixed-model analysis of variance (ANOVA) with Mood (positive vs. neutral) as a between-subjects factor and Time (five MIP ratings) as a within-subjects factor, separately for all four assessments (happiness, pleasantness, sadness, and arousal). Whenever the two-way interaction was significant, it was followed up by independent-sample $t$ tests calculated for each time point to compare the mood levels between the two groups across the experimental session. Additionally, paired-sample $t$ tests between the successive time points for each mood group separately were carried out to assess the strength and direction of the mood change resulting from the MIP. To assess whether imagination abilities or involvement in the (guided-imagery) task differed between groups, a mixed-model ANOVA with Mood as a between-subjects factor and Time (five measurement points) as a within-subjects factor was used.

For the main task, errors from the two no-go trial types (color and orientation) were collapsed (see also Aarts et al., 2012; Aarts \& Pourtois, 2010), whereas only FHs (corresponding to correct and fast-i.e., RTs below the updated cutoff-decisions on go trials) were used as correct responses for comparison purposes with incorrect ones. Accuracy and RT speed were compared between the two mood groups by means of independent-sample $t$ test. The posterror-slowing effect (Laming, 1979; Rabbitt, 1966) was also computed by comparing the two mood groups with a mixed-model ANOVA using Mood (positive vs. neutral) as a between-subjects factor and posttrial Type (hits following errors vs. hits following FHs) as a within-subjects factor.
Analysis of ERPs We analyzed two error-related components: the ERN/Ne and the subsequent Pe component (Falkenstein et al., 2000; Gehring et al., 1993). The ERN is a negative deflection reaching its maximum amplitude over fronto-central electrodes along the midline (electrodes $\mathrm{Fz}$ and FCz), usually peaking $0-100 \mathrm{~ms}$ after (incorrect) response onset. The Pe is the positive deflection following the ERN, and it usually peaks around 150-300 ms post-response-onset, with the maximum amplitude reached over central locations along the midline (electrode $\mathrm{Cz}$ ). On the basis of the electrophysiological properties of the present ERP data set (see Fig. 2a and c), the ERN was defined as the mean amplitude during the 10- to 60-ms postresponse interval at electrode FCz. The Pe was calculated as the mean amplitude during the 145- to 205-ms interval following response onset at electrode Cz. For each ERP component separately, a mixed-model ANOVA was used, with Mood as a between-subjects factor and Accuracy (error vs. FH) as a within-subjects factor. To control for arousal-related effects on these two responselocked ERP components, an additional analysis of covariance (ANCOVA) was calculated with the same experimental factors, including the mean skin conductance level (SCL) as a covariate. To estimate whether the present study was sufficiently powered to detect any group difference, post-hoc G*Power analyses (Faul, Erdfelder, Lang, \& Buchner, 2007) were performed.

Topographical analysis The classical peak analysis outlined above was supplemented by a standard topographical ERPmapping analysis to characterize the topographies (i.e., the actual geometrical configuration of the electric field defined by all 64 channels concurrently) of these two main responselocked ERP components (ERN and Pe), and eventually to assess the effects of positive mood. All these analyses were carried out using the CARTOOL software (Version 3.34; developed by D. Brunet, Functional Brain Mapping Laboratory, Geneva, Switzerland). The basic principles of this method have been described extensively elsewhere (Michel, Seeck, \& Landis, 1999; Murray, Brunet, \& Michel, 2008; Pourtois, Delplanque, Michel, \& Vuilleumier, 2008). First, using $K$ means cluster analysis (Pascual-Marqui, 2002), the dominant topographical maps were identified, using the whole ERP epoch (i.e., from $500 \mathrm{~ms}$ before to $500 \mathrm{~ms}$ after response onset, corresponding to 512 time frames at a $512-\mathrm{Hz}$ sampling rate), including the ERN and Pe components. Next, using spatialfitting procedures, the dominant topographies identified in the preceding step were then fitted back to the individual ERP data/average to determine their expressions across participants and conditions. We used the global explained variance (GEV) as the dependent variable, which corresponds to the goodness of fit of these dominant topographical maps. Finally, these GEV values were entered in an ANOVA with Accuracy and 


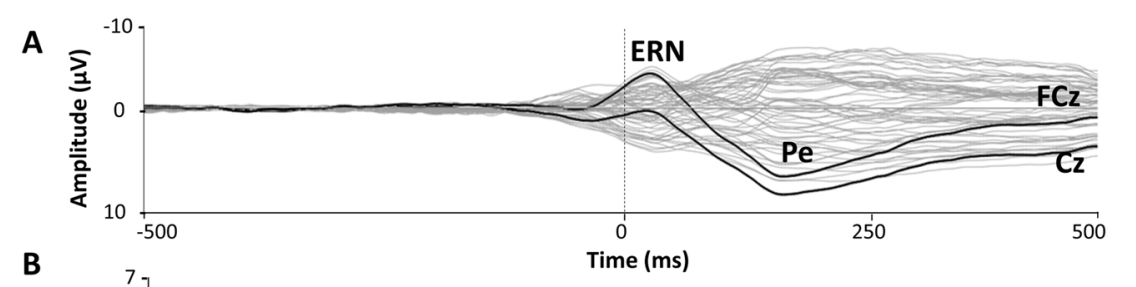

B

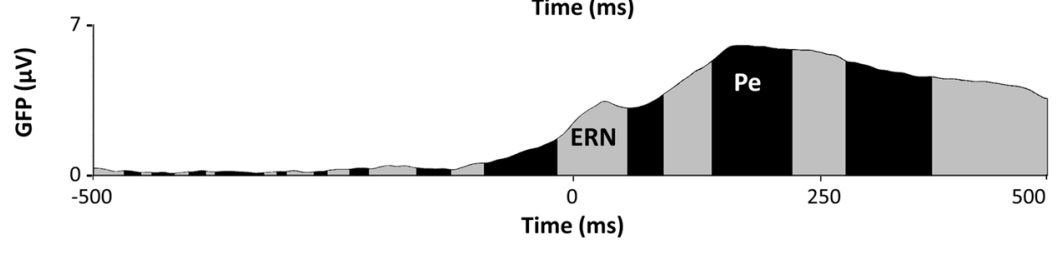

C

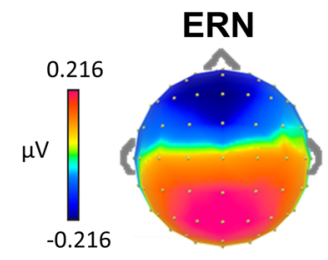

D

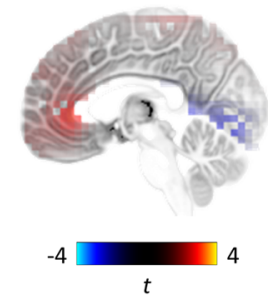

Fig. 2 Main ERP results. a A butterfly view of the grand-average errorrelated ERP data in the neutral mood shows that the ERN reached its maximum around $10-50 \mathrm{~ms}$ at $\mathrm{FCz}$, whereas the subsequent Pe peaked at $145-200 \mathrm{~ms}$ post-response-onset at electrode $\mathrm{Cz}$. The waveforms recorded at $\mathrm{FCz}$ and $\mathrm{Cz}$ are depicted in black. b Results of the corresponding topographical ERP-mapping analysis as a function of global field power (GFP). Two distinct topographical maps, the ERN/ $\mathrm{Ne}$ and $\mathrm{Pe}$, were isolated using a clustering method (see the Method

Map Configuration as within-subjects factors, and Mood as a between-subjects factor.

Source localization To estimate the configuration of the neural generators underlying the previously identified error-related field topographical components, a distributed linear inverse solution was used - namely, standardized low-resolution brain electromagnetic tomography (sLORETA; Pascual-Marqui, 2002). SLORETA solutions are computed within a threeshell spherical head model coregistered to the MNI152 template (Mazziotta et al., 2001). SLORETA estimates the 3-D intracerebral current density distribution within a 5-mm resolution (6,239 voxels, each with an equivalent current dipole). The 3-D solution space is restricted to the cortical gray matter and hippocampus. The head model uses the electric potential field computed with a boundary element method applied to the MNI152 template (Fuchs, Kastner, Wagner, Hawes, \& Ebersole, 2002). Scalp electrode coordinates on the MNI brain are derived from the international $5 \%$ system (Jurcak, Tsuzuki, $\&$ Dan, 2007). The calculation was based on the common
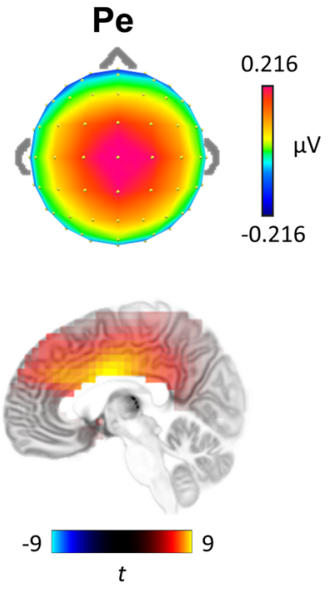

section for details). c These two topographical maps unambiguously correspond to the ERN/Ne and Pe components, respectively. d Using sLORETA, a direct statistical comparison between errors and FHs during the ERN time interval (left panel) shows enhanced error-related activity arising in the rostral part of the ACC, whereas during the Pe time interval, this error-related activity encompasses more dorsal and posterior cingulate regions

average. The inverse-solution results for the ERN and the Pe component were compared between the two mood groups using independent-sample $t$ tests performed on the logtransformed data. We used a stringent nonparametric randomization test (relying on 5,000 iterations) to reveal potential group differences in the inverse-solution spaces through direct statistical comparisons between conditions and mood groups, setting the level of significance for all of the analyses to $p<.01$ (see also Schettino, Loeys, Delplanque, \& Pourtois 2011; Schettino, Loeys, \& Pourtois, 2013).

Analysis of skin conductance EDA was analyzed using Ledalab software V.343 (Benedek \& Kaernbach, 2010a, 2010b), implemented in MATLAB (Version R2014a). The data were smoothed by convolution with an eight-point Gaussian window, and a low-pass Butterworth filter of $5 \mathrm{~Hz}$ was applied. Artifacts were identified and interpolated using visual inspection $(M=1.34 \%, S D=3.02 \%)$. Ledalab returns the SCL as a continuous measure of tonic EDA and separates it from a phasic driver underlying the skin conductance data as 
a continuous measure of phasic EDA or skin conductance responses (SCR). Whereas SCL represents the global electrodermal level, SCR reflects the physiological response to certain events (here with a focus on responses, either correct or incorrect) superimposed on that overall level (Benedek \& Kaernbach, 2010b). The mean SCL for each block and across all blocks (lasting $5 \mathrm{~min}$ ) was calculated per participant. Additionally, phasic SCR was quantified within a response window of 0.5 to $3.5 \mathrm{~s}$ after response onset, and with a minimum amplitude criterion of $0.05 \mu \mathrm{S}$ (Boucsein et al., 2012). Individual data (average phasic driver for each epoch) were range-corrected using the highest and lowest response per participant, following the recommendation of Lykken and Venables (1971), before averaging for each condition. Changes of SCL between the two mood groups were compared using a mixed-model ANOVA with Mood as a betweensubjects factor and Task Block Number $(n=4)$ as a withinsubjects factor. Changes in the SCRs to different responses were also compared using a mixed-model ANOVA with Mood as a between-subjects factor and Accuracy as a within-subjects factor.

\section{Results}

\section{Manipulation checks}

We found a significant interaction of time and mood for all subjective ratings, except for sadness $[F(4,168)=1.88, p=$ $\left..12, \eta^{2}=.040\right]$, happiness $\left[F(4,168)=23.5, p<.001, \eta^{2}=\right.$ $.26]$, pleasantness $\left[F(4,168)=9.80, p<.001, . \eta^{2}=.17\right]$, and arousal $\left[F(4,168)=7.69, p<.001, \eta^{2}=.15\right]$. No significant group differences in mood ratings were found at baseline, prior to the first MIP, for any of the different ratings used [all $t \mathrm{~s}(42) \leq 1.57, p \geq .10$ ]. After the MIP, only the positive mood group showed increased levels for happiness, pleasantness, and arousal relative to baseline $[t \mathrm{~s}(21) \geq 3.3, p \mathrm{~s} \leq .003]$, as well as higher levels of happiness, pleasantness, and arousal relative to the neutral mood group for all successive time points [all $t \mathrm{~s}(42) \geq 3.3, p \mathrm{~s}<.020]$.

\section{Behavioral results}

Task performance was similar between the two groups (see Table 2): Error rates were not different between them $[t(42)=$ $0.11, p=.91, d=0.034]$. Likewise, the ratios between fast and slow hits were similar [t(42) $=0.89, p=.38, d=0.027]$. Moreover, the correlations between speed and accuracy were also balanced between the happy and neutral mood groups $\left[r_{\mathrm{Neu}}(20)=-.72, p<.001 ; r_{\mathrm{Hap}}(20)=-.54, p=.009 ; Z=0.95\right.$, $p=.34]$. The groups did not differ in RT speed, either (for any of the trial types considered; see Table 2), nor in the individual time limits used to demarcate FHs from SHs $[t(42)=0.027, p=.978$,
Table 2 Accuracy and mean reaction times per mood group for each trial type

\begin{tabular}{lcccccccc}
\hline & \multicolumn{1}{c}{ Neutral } & \multicolumn{1}{c}{ Happy } & \multicolumn{3}{c}{ Comparison } \\
\hline \multicolumn{2}{l}{ Trial Type } & $M$ & $S D$ & $M$ & $S D$ & $t(42)$ & $p$ & $d$ \\
\multicolumn{2}{l}{ Numbers of Trials } & per Condition & & & & & \\
Fast hit & 98.3 & 30.2 & 93.9 & 35.6 & 0.45 & .65 & .12 \\
Error & 50.4 & 24.7 & 49.6 & 23.3 & 0.11 & .91 & .001 \\
Speeds (ms) per Condition & & & & & & \\
Fast hit & 217 & 34.3 & 215 & 24.2 & 0.20 & .84 & .06 \\
Error & 314 & 44.6 & 321 & 36.0 & 0.20 & .84 & .17 \\
Post-fast-hit & 258 & 36.0 & 259 & 29.2 & 0.16 & .87 & .05 \\
Posterror & 275 & 52.8 & 283 & 32.5 & 0.58 & .57 & .17 \\
Posterror slowing & 15.8 & 32.3 & 21.6 & 20.1 & 0.71 & .48 & .21 \\
\hline
\end{tabular}

$d=0.001]$. A classical posterror slowing effect was observed. RTs were longer for hits after an error than for hits following an FH $\left[F(1,42)=22.1, p<.001, \eta^{2}=.35\right]$. However, the magnitude of the posterror slowing effect was not influenced by mood [main effect of mood, $F(1,42)=0.49, p=.49, \eta^{2}=.008$; interaction of mood and accuracy, $\left.F(1,42)=0.18, p=.67, \eta^{2}=.004\right]$.

A manipulation check of the MIP did not reveal any significant group differences in imagination abilities $[F(1,42)=$ $\left.0.06, p=.807, \eta^{2}<.001\right]$. This null finding therefore suggests that both groups were equally strongly involved in the visual imagery, thereby ruling out a strong asymmetry between them regarding (cognitive) load or efforts made to actively relive the targeted memory.

\section{ERP results}

The analysis performed on the mean ERN amplitudes at electrode $\mathrm{FCz}$ showed a significant main effect of accuracy $[F(1$, $\left.42)=45.33, p<.001, \eta^{2}=.51\right]$, but no significant effects of $\operatorname{mood}\left[F(1,42)=1.34, p=.25, \eta^{2}<.001\right]$ or interaction between these two factors $\left[F(1,42)=1.33, p=.26, \eta^{2}=\right.$ .016]. The amplitude of the ERN (for errors) was larger than the amplitude of the correct-related negativity (CRN, for correct responses); see Fig. 3a. Entering the mean SCL as a covariate revealed no significant effect $[F \mathrm{~s}(1,41) \leq 1.33, p \mathrm{~s} \geq$ $\left..26, \eta^{2} \mathrm{~s} \leq .031\right]$. Post-hoc power estimations confirmed that the present study was sufficiently powered to detect a group difference at the ERN level $(1-\beta=.89)$.

For the Pe component, recorded at electrode $\mathrm{Cz}$, the analysis showed a significant main effect of accuracy $[F(1,42)=75.99, p$ $\left.<.001, \eta^{2}=.62\right]$. Importantly, the main effect of $\operatorname{mood}[F(1,42)$ $\left.=8.74, p=.005, \eta^{2}=.17\right]$ and the interaction between these two factors $\left[F(1,42)=4.13, p=.049, \eta^{2}=.034\right]$ were also significant. When the mean SCL was added as a covariate, the main effect of $\operatorname{mood}\left[F(1,41)=8.52, p=.006, \eta^{2}=.17\right]$ and the interaction between accuracy and $\operatorname{mood}[F(1,41)=4.50, p=$ $\left..040, \eta^{2}=.092\right]$ remained significant. The Pe amplitude for 

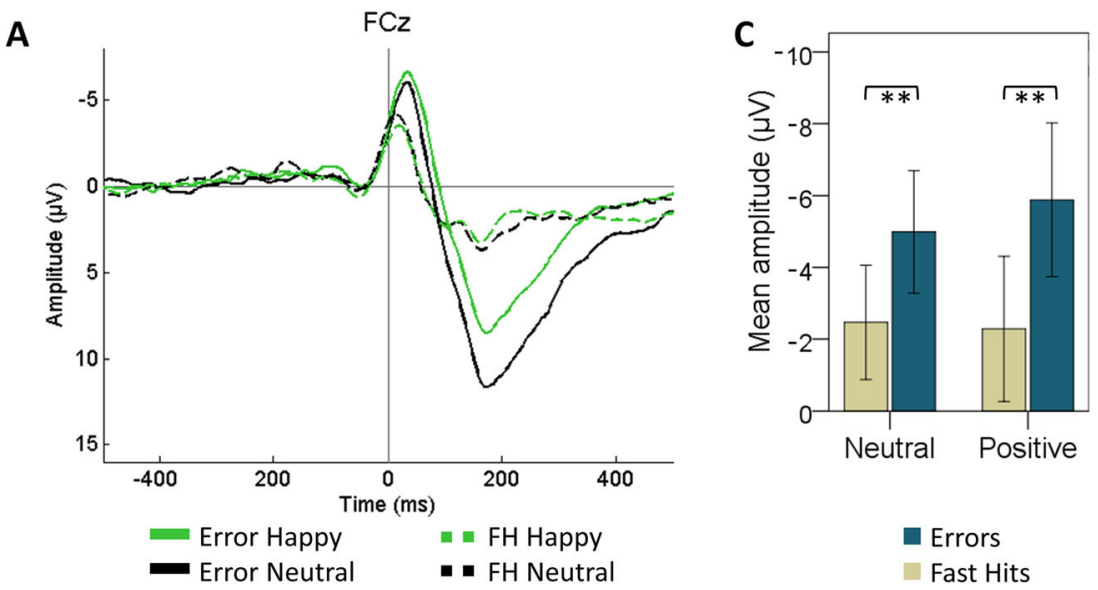

B

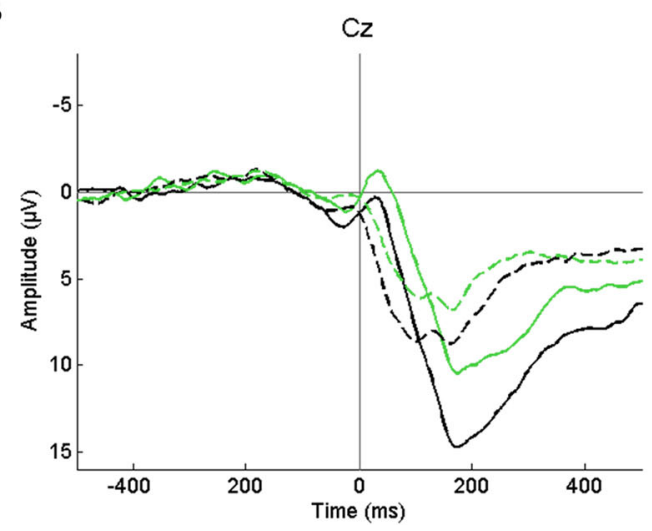

D

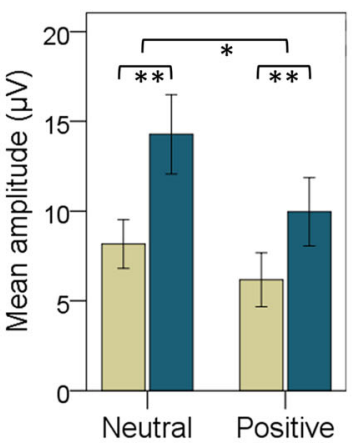

Fig. 3 ERP results. a Grand-average ERP waveforms at FCz (ERN), shown for each mood group (neutral and happy) and response type (error and $\mathrm{FH}$ ) separately. b Grand-average ERP waveforms at $\mathrm{Cz}(\mathrm{Pe})$, shown for each mood group (neutral and happy) and response type (error and $\mathrm{FH}$ ) separately. c Mean amplitudes at $\mathrm{FCz}$ (expressed in microvolts) for the ERN (errors) and the CRN (hits) for the two groups separately. A significant main effect of accuracy was found whereby the ERN was

larger than the CRN, equally in the two mood groups. d Mean amplitudes at $\mathrm{Cz}$ (expressed in microvolts) for the Pe (errors) and $\mathrm{Pc}$ (correct hits) for the two groups separately. Unlike the ERN, the Pe was reliably reduced in the happy mood group, as revealed by a significant Group $\times$ Accuracy interaction. The error bar represents the $95 \%$ confidence intervals (CIs). ${ }^{*} p<.05,{ }^{* * *} p<.001$

errors was larger than the amplitude of the positivity related to correct responses $(\mathrm{Pc})$ in both groups [neutral, $t(21)=7.23, p<$ $.001, d=1.48$; happy, $t(21)=4.99, p<.001, d=0.98]$, but this difference was reduced in the happy group. More specifically, whereas the Pc only trended toward being lower for happy than for neutral participants $[t(42)=2.04, p=.047, d=0.61]$, the Pe was clearly blunted in the happy as compared to the neutral mood group $[t(42)=3.07, p=.004, d=0.93]$; see Fig. $3 b$. Post-hoc power estimations confirmed that the present study was sufficiently powered to detect a group difference at the $\mathrm{Pe}$ level $(1-\beta=.99)$.

\section{Skin conductance results}

The ANOVA performed on the SCL values did not reveal any significant change of tonic arousal during the experiment [main effect of task block: $\left.F(3,126)=0.79, p=.50, \eta^{2}=.019\right]$ or any difference between the two mood groups $[\operatorname{mood}, F(1,42)=$
$0.017, p=.89, \eta^{2}<.001$; task block by group interaction, $F(1$, 126) $\left.=0.11, p=.96, \eta^{2}=.003\right]$. However, an analysis performed on the phasic SCR to either errors or FHs did reveal a significant difference between these opposite response types $[F(1,42)=$ $\left.6.44, p=.015, \eta^{2}=.14\right]$, with, as expected, a higher SCR for errors than for FHs $\left[t(43)=2.56, p=.014, d=0.29 ; M_{\mathrm{FH}}=.068\right.$, $\left.S D_{\mathrm{FH}}=.059 ; M_{\mathrm{Err}}=.091, S D_{\mathrm{Err}}=.096\right]$. The $\mathrm{SCR}$ was not globally influenced by $\operatorname{mood}\left[F(1,42)=0.44, p=.51, \eta^{2}=\right.$ $.01]$, and the interaction between mood and accuracy was not significant $\left[F(1,42)=0.13, p=.72, \eta^{2}=.001\right]$.

\section{Topographical mapping results}

A solution with seven dominant maps explained $99.0 \%$ of the variance. During the time interval of the ERN (10-60 ms postresponse-onset), a main topographical change between errors and FHs was evidenced. Although the topography of the ERN (errors) was qualified by a clear negative deflection at fronto- 
central electrode positions (around FCz), the CRN map (FHs) was characterized by a weaker and broader prefrontal negative deflection (see Aarts \& Pourtois, 2010; Aarts et al., 2013, for similar results with the same task); see Fig. $2 \mathrm{~b}$ and c.

The ANOVA run on the GEV values obtained for each component (ERN/CRN) revealed a significant interaction between accuracy and map configuration $[F(1,42)=20.41, p<$ $\left..001, \eta^{2}=.13\right]$. Whereas the CRN map explained more variance for FHs than for errors $[t(43)=4.89, p<.001, d=0.86]$, the exact opposite pattern was found for the ERN map $[t(43)=$ $3.57, p<.001, d=0.56]$. This effect was not modulated by mood, however [mood, $F(1,42)=3.52, p=.068, \eta^{2}=.07$; interactions with mood, $\left.F \mathrm{~s}(1,42)<0.62, p \mathrm{~s}>.44, \eta^{2} \mathrm{~s}<.01\right]$; see Fig. $4 a$ and $b$.

During the Pe time interval (145-205 ms post-responseonset), a specific error-related topography was likewise evidenced. It was characterized by a large positivity surrounding the $\mathrm{Cz}$ electrode position (Pe), whereas FHs elicited a weaker and broader posterior positivity (Pc). Consistent with the observation that mood influenced the Pe when considering the amplitude of this component at electrode $\mathrm{Cz}$, the ANOVA run on the GEV values obtained after fitting (hence, reflecting the topography of this mid-latency error-related ERP component) revealed a significant interaction between accuracy, map, and
$\operatorname{mood}\left[F(1,42)=4.61, p=.038, \eta^{2}=.03\right]$. As can be seen in Fig. 4, the variance of the topography elicited by FHs in the neutral group could be explained better by the topographical map of the Pc $[t(21)=3.56, p=.002, d=0.97]$, but error variance in that group could be explained better by the Pe topographical map $[t(21)=4.40, p<.001, d=1.03]$, whereas in the happy group, there was no such differentiation for the Pe map $[t(21)=1.77, p=.091, d=0.43]$, but there was for the topographical map of the $\operatorname{Pc}[t(21)=2.31, p=.031, d=0.35]$; see Fig. $4 \mathrm{c}$ and d.

\section{Source localization results}

The statistical comparison in the inverse-solution space between errors and FHs within the time window of the ERN/ CRN (10-60 ms post-response-onset) revealed widespread clusters with stronger activation for errors than for FHs: one located within the midcingulate/anterior cingulate cortex (ACC) [including Brodmann areas (BAs) 24, 32; max. at 5x, $40 y, 5 z$ in BA $32, t(43)=2.98, p=.003]$ and another one corresponding to the left frontal gyrus (FG) [including BAs $7-11$, max. at $-15 x, 50 y, 10 z$ in BA 7 ; $t(43)=2.63, p=.007]$; see Fig. 2d. However, mood did not influence these effects [group comparisons for errors: at ACC, $t(42)=0.16, p=.44$;
A

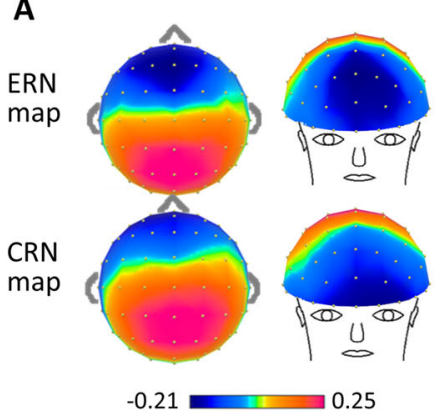

$\mu \mathrm{V}$

C

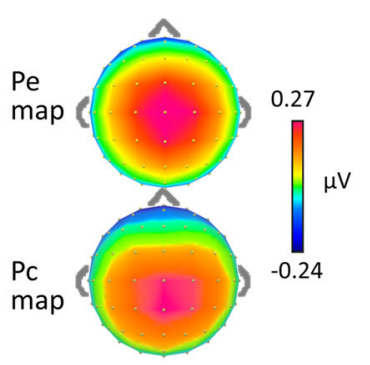

B

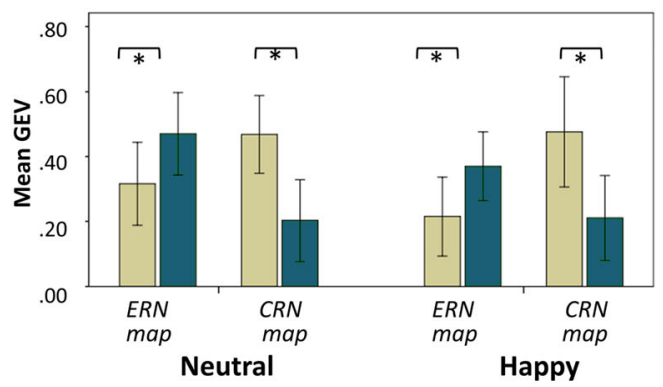

D

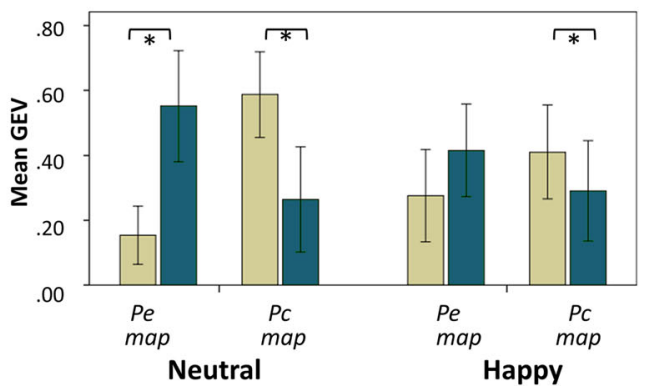

Fig. 4 Results of the topographical ERP-mapping analysis. a The scalp map of the ERN shows negative activity over prefrontal electrodes along the midline, whereas the CRN has a qualitatively different scalp configuration. b For both groups, the ERN/Ne topographical component explains more variance for errors than for hits, and the topographical component corresponding to the CRN shows the reverse pattern. $\mathbf{c}$ The scalp map of the Pe is characterized by broad positive

activity over central electrode positions, whereas FHs are associated with a qualitatively different scalp configuration during the same time interval (Pc). d The Pe topographical component explains more variance for errors than for FHs, but in the neutral group only (this effect is substantially attenuated in the happy mood group), whereas the Pc component explains more variance for FHs than for errors in both groups. Error bars correspond to $95 \%$ CIs. ${ }^{*} p<.05$ 
at FG, $t(42)=0.38, p=.35$; group comparisons for FHs: at ACC, $t(42)=0.40, p=.34$; at FG, $t(42)=0.35, p=.36]$.

During the time interval corresponding to the Pe component (145-205 ms post-response-onset), the statistical comparison between errors and FHs showed that errors led to stronger activation in a broad cluster extending from anterior/posterior parts of the cingulate gyrus [including BAs 23, 24, 30-32; max. at $5 x,-10 y, 30 z$, in BA $24 ; t(43)=8.0, p<.001]$ to frontal (including BAs 2-7, 18, 19, 37, 40) and parietal (including BAs 8-11, 20-22, 39) regions. Furthermore, a bilateral cluster was found within the insula with stronger activation for errors than for FHs [BA 13, max. at $-35 x,-25 y, 20 z ; t(43)=7.89, p<$ $.001]$; see Fig. 2d. Importantly, a direct statistical comparison between the two mood groups for errors confirmed an alteration of the intracranial generators giving rise to the Pe: In the happy mood group, decreased activations (relative to the neutral mood group) were observed within the posterior part of the cingulate cortex, spreading to superior frontal and parietal gyrus [including BAs 3-6, 8, 24, 31; max. at $-5 x,-10 y, 70 z$ in BA $6 ; t(42)=$ $4.13, p<.001$ ], as well as the insula bilaterally [with an effect more pronounced in the right hemisphere, max. at $45 x,-15 y$, $15 z$ in BA $13 ; t(42)=3.29, p<.001]$. By comparison, only very few nodes in the posterior parietal cortex showed a small difference between the two mood groups for FHs [max at $-20 x$, $55 y, 70 z$ in BA $7 ; t(42)=2.79, p=.007]$; see Fig. 5 .
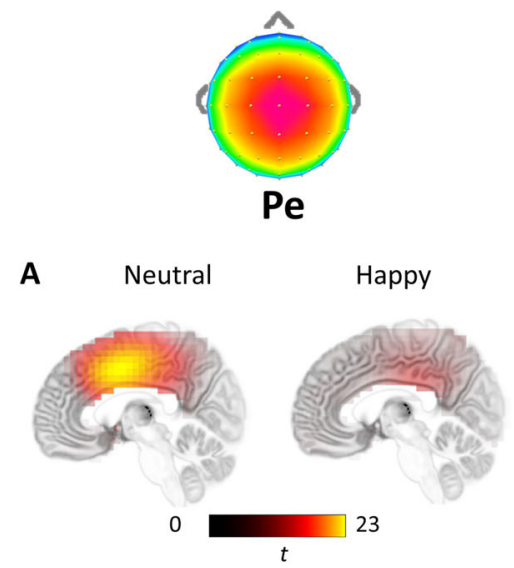

C
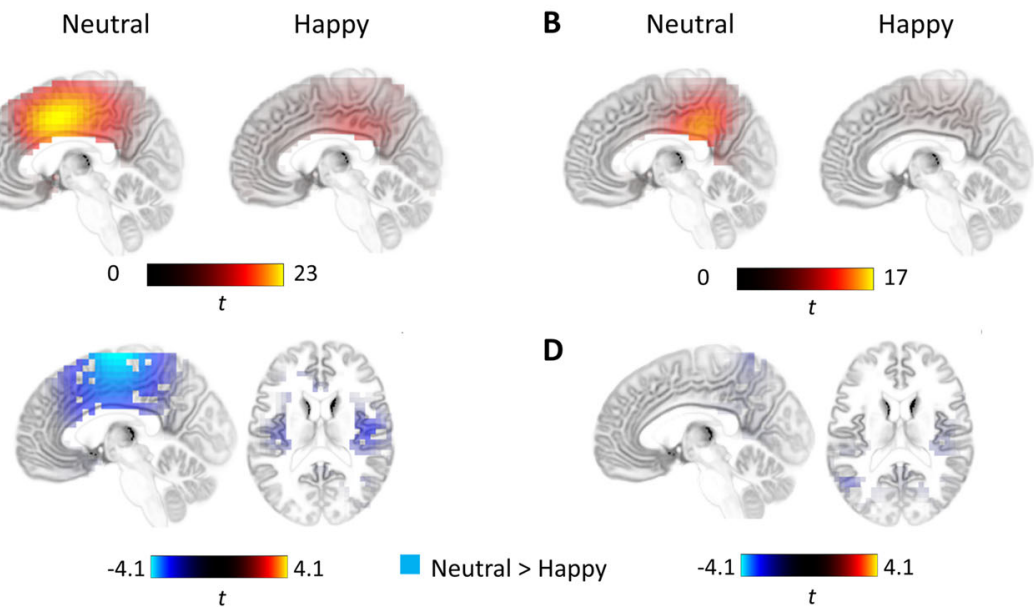

D

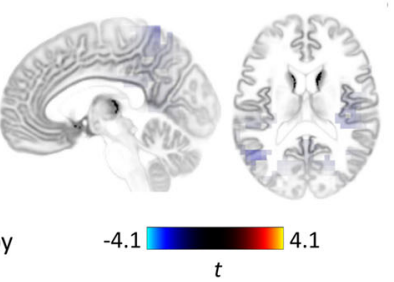

parts of the cingulate gyrus (BA 31), which is less active in the latter group. c A direct statistical comparison between the two groups for errors reveals that the neutral group had stronger activations than the happy group within the dorsal ACC and posterior cingulate gyrus (BAs $24,31,6$ ), as well as within the insula bilaterally (BA 13). d For FHs, group differences (in these same regions) are clearly more modest and circumscribed than for errors 
level) or internalizing traits and psychopathology (with which the ERN is usually found to be overactive, whereas the subsequent Pe is decreased, as compared to neutral mood; see Koban \& Pourtois, 2014; Weinberg, Kotov, \& Proudfit, 2015). Our new results show that positive mood selectively decreases the Pe component, while leaving the preceding ERN/Ne component unchanged (relative to an active control condition with neutral mood content), suggesting a component-specific effect triggered by the happy mood state during error monitoring. Importantly, this effect was evidenced in the absence of obvious differences at the behavioral level between the two mood groups for both task performance and posterror adaptation. Likewise, arousal did not differ between the two groups. Moreover, by using complementing topographical and source localization methods, we could gain insight into the actual neurophysiological expression of this selective change at the Pe level, as well as the underlying neural sources likely giving rise to it. Below, we discuss the implications of these new results in greater detail.

The MIP used in this study led to the elicitation of a specific mood or emotional state characterized by a high level of experienced happiness and pleasure (positive emotion dimension), while leaving sadness (as a negative affective dimension) unchanged. Moreover, we found that this manipulation gave rise to an interesting dissociation between arousal at the subjective level (which was increased in the happy relative to the neutral mood group; see also Bakic et al., 2015) and tonic activity as measured at the autonomic nervous system (ANS) level using SCL (which was not different between the two mood groups). Importantly, the lack of an SCL difference between the two mood groups could not be explained by the absence of normal and differential ANS reactions detected in our participants, since they did respond stronger to response errors than to FHs, as captured by the concurrent SCR measurement. However, the lack of a group difference in tonic activity (SCL) should be interpreted with caution in the present case, because the experimental design and task demands we used may have obscured a systematic change in SCL with happy mood. One factor likely accounting for this dissociation pertains to the way that the measurement was made: Whereas the subjective ratings were performed immediately after the mood induction, the (objective) SCL was defined as the mean throughout the task. It has been shown previously that induced arousal, but not valence, decreases over time (Gomez, Zimmermann, Schär, \& Danuser, 2009), which has been explained as a down-regulation of physiological arousal that interferes with task performance. At any rate, it appears plausible to conclude that the specific happy mood state elicited by the MIP in our study was not unspecific or undifferentiated, but instead likely corresponded to genuine joy or pleasure (i.e., a state of well-being characterized by contentment), as opposed to other positive mood states, such as bliss or euphoria, or conversely to serenity or ataraxis, for which arousal- related changes in the ANS are likely to be observed (Christie \& Friedman, 2004; Shiota, Neufeld, Yeung, Moser, \& Perea, 2011). As a limitation, we note, however, that because participants reported increased levels of both happiness and pleasantness following the MIP, the specific mood induced probably lacked clear differentiation in terms of the positive emotion content experienced. Nevertheless, our attempt to specify the actual mood state elicited by the MIP, based on both subjective ratings and objective (psychophysiological) measurements, is important, because positive emotion or affect is usually not conceived as a unitary construct, but likely encompasses different forms or expressions (ranging from astonishment to euphoria), each of which is susceptible to influencing cognition, physiological responding, motivation, or behavior in a specific way (Shiota et al., 2014). In fact, as our behavioral results clearly show, the elicited joy in the happy mood group did not interfere with cognitive control or inhibition "directly" (as well as with posterror adjustments), since behavioral performance was matched between the two mood groups (see Vanlessen et al., 2015, for a similar conclusion). This observation is important because it confirms that the idiosyncratic joy or contentment experienced by the participants (in the happy mood group) was not merely bringing noise or distraction (Dreisbach \& Goschke, 2004), but it did alter the subjective experience of specific events - namely response errors - as revealed by the corresponding ERP results. From a methodological point of view, the balanced behavioral performance between the two mood groups is valuable because the differential error monitoring seen at the ERP level between them can therefore not be accounted for by asymmetries in the numbers of response errors or the speed with which they were committed, two factors that reliably influence the shape and morphology of response-locked ERP components, especially the ERN/NE (Gehring et al., 1993; Olvet \& Hajcak, 2009). Also note that the lack of group differences at the behavioral level (speed and accuracy) was not odd in the present case, but was expected given the specifics of the go/no-go task used. Since the RT deadline was calibrated and updated at the single-participant level, it inevitably led to comparable numbers of response errors (and balanced speed) between the two groups, as had already been reported in previous studies using the same task and between-subjects experimental design (see Aarts \& Pourtois, 2010; Aarts et al., 2013; Koban, Brass, Lynn, \& Pourtois, 2012; Rigoni, Pourtois, \& Brass, 2015; Walentowska, Moors, Paul, \& Pourtois, 2016).

A novel and important result of our study is that, despite the balanced behavioral performance between the two mood groups, the experience of joy did influence error monitoring, in a component-specific fashion. Although the ERN components were similar between the groups - that is, early error detection mechanisms remained impermeable to positive mood - the Pe component was reliably diminished in the 
happy as compared to the neutral mood group. This effect was visible at the scalp level using both standard component/peak measurements, as well as a complementing topographical ERP-mapping analysis. Furthermore, we found by using source localization methods that this effect was likely caused by decreased activation in a network comprising the posterior cingulate and insular cortices. The contribution of these specific brain regions to the generation of the Pe component had been shown previously (Dhar, Wiersema, \& Pourtois 2011; Herrmann, Römmler, Ehlis, Heidrich, \& Fallgatter, 2004; Mathewson, Dywan, \& Segalowitz, 2005; Van Veen \& Carter, 2002). Several authors have put forward the notion that the Pe component might reflect the processing of the motivational significance of response errors (Leuthold \& Sommer, 1999; O'Connell et al., 2007; Overbeek et al., 2005; Ridderinkhof et al., 2009; Ullsperger, Harsay, Wessel, \& Ridderinkhof, 2010). In this context, errors are considered salient events (because they are deviant and usually worsethan-expected events), eliciting an "automatic" orienting response (Notebaert et al., 2009) and activating a "salience network" in which the anterior insular cortex (and its reciprocal anatomical connections with the ACC) plays a critical role (Inzlicht, Bartholow, \& Hirsh, 2015; Seeley et al., 2007; Uddin, 2014; Ullsperger et al., 2010). For example, error monitoring was found to be heightened at the Pe level selectively during placebo analgesia (Koban et al., 2012), suggesting that this specific state likely increases the motivational significance of errors, whereas happy mood conversely appears to decrease it. In light of this evidence, it is therefore plausiblebut somewhat speculative at this stage - to assume that the experience of joy or contentment could very well transiently decrease the otherwise heightened salience usually associated with error commission. Importantly, we can rule out the possibility that this effect resulted from a dampened reaction to response errors in happy relative to neutral participants in general. First, posterror slowing, which is thought to reflect unspecific attention orienting to (deviant) response errors (Notebaert et al., 2009), and which is increased by (subjective) arousal (De Saedeleer \& Pourtois, 2016), was preserved in the happy mood group. Second, the ANS reaction to errors, as captured by the SCR, was also preserved in happy participants. Third, when controlling statistically for changes in SCL (tonic arousal) using an ANCOVA, the Pe component to response errors was still found to be reliably blunted in the happy as compared to the neutral mood group. Therefore, we conjecture that the experience of joy/ contentment in adult healthy participants likely alters the subjective evaluation of response errors (and more specifically, their perceived salience) rather than the arousal or ANS reaction to them. When considering the assumption of mood congruency effects (Rusting, 1998; Sharot, Korn, \& Dolan, 2011; Tamir \& Robinson, 2007), it is therefore possible that the happy mood elicited in our study did "shield" participants from negative (mood-incongruent) information, such as response errors. This could be achieved by down-regulating their salience or meaning (at the Pe level), thereby fostering the pursuit and maintenance of the (pleasant) mood state currently being experienced by the participant. Hence, happy mood could provide participants with an adaptive mechanism that seeks to conserve the benefits associated with the current mood state, which has been related to building up additional resources and protecting an individual from the experience of stress or negative affect (Fredrickson, 2004; Schwarz \& Bless, 1991). In such a state of joy or contentment, there is presumably no need to enhance or trigger alertness to response errors, since the environment in which they happen is regarded as "safe," and errors therefore forfeit their negative motivational significance or salience. Because this Pe effect related to the induction of happy mood was observed in the absence of any change at the behavioral level (as compared to the control condition, with a neutral mood content), and since no direct evidence was provided concerning a possible change in the actual motivational or emotional processing of errors with happy mood in this study, further research will be needed to corroborate this assumption more directly. In this context, the use of priming methods meant to explore the motivational or emotional value of actions, including errors (see, e.g., Aarts et al., 2012), might be valuable.

The observation of a component-specific effect during error monitoring triggered by joy or contentment, at the Pe level, and the direction of this neurophysiological effect (viz., a reduced Pe amplitude) are worth discussing further. Interestingly, previous ERP studies had already reported decreased Pe amplitudes during error monitoring among participants with depression or trait-related negative affect (Aarts et al., 2013; Alexopoulos et al., 2007; A. J. Holmes \& Pizzagalli, 2010; Olvet et al., 2010; Schrijvers et al., 2009; Schroder et al., 2013), which can be considered - with some reservation, since trait and state effects do not always produce comparable changes in $\mathrm{PM}$ - as the opposite mood state from the emotion (transiently) experienced in the happy mood group in this study, yet with similar electrophysiological effects seen in both cases. Although puzzling, these similar neurophysiological effects found in two opposite mood states could actually reflect different underlying processes or mood-dependent alterations in these two cases. Whereas in the case of depression (and trait negative affect), a reduced Pe is often interpreted as reflecting an inability to adapt or change cognitive control functions in a timely manner in response to negative events (or perhaps reflecting impairments to consciously register them; see Frank, D'Lauro, \& Curran, 2007; Hajcak, McDonald, \& Simons, 2003b; Nieuwenhuis et al., 2001), such an interpretation appears difficult to hold in the case of happy mood and positive emotions, given that they usually promote (rather than undermine) creativity and flexibility, and perhaps even augment cognitive control in specific circumstances (Fredrickson, 2004; Nadler et al., 2010). 
Interestingly, our new findings are also compatible with an earlier ERP study showing decreased Pe amplitudes with relaxed mood (following a social meal; Sommer, Stürmer, Shmuilovich, Martin-Loeches, \& Schacht, 2013), suggesting that a reduced Pe with positive affect (conceived as a state) could be observed across different contexts or task settings. More generally, our new ERP results cast doubt on the assumption that a reduced Pe during error monitoring necessarily denotes decreased cognitive control during error monitoring and/ or is a (neurophysiological) landmark of negative affect. Similarly, the lack of modulation of the ERN with positive mood in our study is also informative, given that amplitude variations in this early error-related component have often been linked in the past to negative affect and internalizing traits or disorders (Hajcak et al., 2003b, 2004; Luu et al., 2000; Wiswede, Münte, Goschke, \& Rüsseler, 2009). We had previously reported an enhanced ERN amplitude in a positive mood state, when errors were embedded in a reinforcement-learning context (Bakic et al., 2014). However, in this study errors likely acquired a different meaning than in the present case, in which they rather reflected attentional lapses or a breakdown of impulse control. Hence, it appears that positive mood is versatile and can produce different effects during error monitoring, depending on specific contextual or situational cues or task demands (Huntsinger, 2012). It can either increase (at the ERN level) reward prediction error when errors serve as potent learning signals (Bakic et al., 2014) or, alternatively, lower their motivational significance or salience (at the Pe level) when they provide clear challenges to self-efficacy, as was found in our present study.

Some limitations warrant comment. First, although sLORETA is an empirically well supported source localization technique (Mulert et al., 2004; Pizzagalli et al., 2004; Zumsteg, Friedman, Wennberg, \& Wieser, 2005), the inverse solutions obtained should be interpreted with caution, because these mathematical reconstructions necessarily remain imprecise and suffer from low spatial resolution. Second, because the Pe was previously associated with error awareness (see Nieuwenhuis et al., 2001; Ullsperger et al., 2010), changes not only in error detection or monitoring, but also in error awareness as a function of happy mood, should be evaluated more systematically in the future. The reduced Pe component found in the happy mood group in our study is unlikely to be explained by a change in error awareness in this group, however, because almost all response errors (99\%) are usually consciously detected by all participants in this speeded go/ no-go task (see Vocat et al., 2008), and normal posterror adaptation was found in both groups in the present case. Third, we observed a relatively large CRN component in our study. However, this result was not unexpected, but very much in line with previous ERP studies using the same speeded go/nogo task with very strict time pressure and an updated RT deadline (Vocat et al., 2008). These conditions necessarily increased uncertainty at the time of keypress, given that performance was based on both accuracy and speed (Walentowska et al., 2016). As a matter of fact, (enhanced) uncertainty also increases the CRN component (Coles et al., 2001; Falkenstein et al., 2000; Gehring et al., 1993). Accordingly, it remains to be tested whether effects of (positive) mood might also be observed at the ERN level, when uncertainty (regarding accuracy and/or speed) is kept low. Finally, although we tried to measure and control levels of arousal in the two mood groups, subjective arousal was still larger after the MIP in the happy than in the neutral mood group (despite the use of physical-activity-related memories during guided imagery in the latter group), whereas the objective arousals (skin conductance as a measure of automatic arousal) were comparable between the two groups. Even though arousal was unlikely to explain the modulation of the Pe component with happy mood in our study (see here and in the Results section), future studies will be needed to assess the specific contributions of (subjective and objective) arousal versus valence (during the experience of a specific mood state) to error-monitoring brain functions.

In conclusion, our results show that an emotional state induced and characterized by joy or contentment can reliably alter, and presumably lower, the motivational significance or salience of response errors inadvertently committed during a speeded go/no-go task, with effects visible at the Pe level selectively (as opposed to the preceding ERN/Ne component, which remained impermeable to these mood changes). This neurophysiological effect, which did not correspond simply to a blunted arousal reaction to errors with happy mood, likely stemmed from reduced activation in the anterior insula and posterior cingulate cortex (as confirmed by complementing source localization results), which were presumably both involved in processing of the salience of these worse-thanexpected events. Therefore, we conclude that the transient experience of joy or contentment in healthy adult participants does not interfere with cognitive control, inhibition, or automatic error detection (reflected by the ERN component or concurrent changes in the SCR), but instead appears to enable an adaptive and mood-congruent change in the organism such that the (negative) meaning or impact of these unwanted events is transiently downplayed.

Author note K.P. is funded by a $\mathrm{PhD}$ fellowship from the Research Foundation Flanders (FWO). The university position of W.W. was supported by funding from Jagiellonian University within the "SocietyEnvironment-Technology (SET)" project (S/FS0/0117.30) and a Mobility Plus III grant (1096/MOB/2013/0), financed by the Polish Ministry of Science and Higher Education. T.D. received a grant from the Foundation Pierre Deniker, Paris. This work is also funded by grants from the FWO and the special research fund (Ghent University), awarded to G.P. We thank three anonymous reviewers for the valuable comments made on an earlier version of the manuscript. The authors declare that the research was conducted in the absence of any commercial or financial relationships that could be construed as a potential conflict of interest. 


\section{References}

Aarts, K., De Houwer, J., \& Pourtois, G. (2012). Evidence for the automatic evaluation of self-generated actions. Cognition, 124, 117-127. doi:10.1016/j.cognition.2012.05.009

Aarts, K., \& Pourtois, G. (2010). Anxiety not only increases, but also alters early error-monitoring functions. Cognitive, Affective, \& Behavioral Neuroscience, 10, 479-492. doi:10.3758/CABN.10.4.479

Aarts, K., Vanderhasselt, M. A., Otte, G., Baeken, C., \& Pourtois, G. (2013). Electrical brain imaging reveals the expression and timing of altered error monitoring functions in major depression. Journal of Abnormal Psychology, 122, 939-950. doi:10.1037/a0034616

Alexopoulos, G. S., Murphy, C. F., Gunning-Dixon, F. M., Kalayam, B., Katz, R., Kanellopoulos, D., \& Foxe, J. J. (2007). Event-related potentials in an emotional go/no-go task and remission of geriatric depression. NeuroReport, 18, 217-221. doi:10.1097/WNR.0b013 e328013ceda

Bakic, J., De Raedt, R., Jepma, M., \& Pourtois, G. (2015). What is in the feedback? Effect of induced happiness vs. sadness on probabilistic learning with vs. without exploration. Frontiers in Human Neuroscience, 9(584), 1-13. doi:10.3389/fnhum.2015.00584

Bakic, J., Jepma, M., De Raedt, R., \& Pourtois, G. (2014). Effects of positive mood on probabilistic learning: Behavioral and electrophysiological correlates. Biological Psychology, 103, 223-232. doi:10.1016/j.biopsycho.2014.09.012

Benedek, M., \& Kaernbach, C. (2010a). A continuous measure of phasic electrodermal activity. Journal of Neuroscience Methods, 190, 80 91. doi:10.1016/j.jneumeth.2010.04.028

Benedek, M., \& Kaernbach, C. (2010b). Decomposition of skin conductance data by means of nonnegative deconvolution. Psychophysiology, 47, 647-658. doi:10.1111/j.1469-8986.2009.00972.x

Botvinick, M. M., \& Braver, T. (2015). Motivation and cognitive control: From behavior to neural mechanism. Annual Review of Psychology, 66, 83-113. doi:10.1146/annurev-psych-010814-015044

Boucsein, W., Fowles, D. C., Grimnes, S., Ben-Shakhar, G., Roth, W. T., Dawson, M. E., \& Filion, D. L. (2012). Publication recommendations for electrodermal measurements. Psychophysiology, 49, 1017 1034. doi:10.1111/j.1469-8986.2012.01384.x

Bradley, M., \& Lang, P. J. (1994). Measuring emotion: The SelfAssessment Manikin and the semantic differential. Journal of Behavior Therapy and Experimental Psychiatry, 25, 49-59. doi:10.1016/0005-7916(94)90063-9

Chiu, P. H., \& Deldin, P. J. (2007). Neural evidence for enhanced error detection in major depressive disorder. American Journal of Psychiatry, 164, 608-616. doi:10.1176/appi.ajp.164.4.608

Christie, I. C., \& Friedman, B. H. (2004). Autonomic specificity of discrete emotion and dimensions of affective space: A multivariate approach. International Journal of Psychophysiology, 51, 143153. doi:10.1016/j.ijpsycho.2003.08.002

Coles, M. G., Scheffers, M. K., \& Holroyd, C. B. (2001). Why is there an $\mathrm{ERN} / \mathrm{Ne}$ on correct trials? Response representations, stimulusrelated components, and the theory of error-processing. Biological Psychology, 56, 173-189. doi:10.1016/S0301-0511(01)00076-X

De Saedeleer, L., \& Pourtois, G. (2016). Evaluative priming reveals dissociable effects of cognitive vs. physiological anxiety on action monitoring. Emotion, 16, 498-514. doi:10.1037/emo0000149

Dhar, M., Wiersema, J. R., \& Pourtois, G. (2011). Cascade of neural events leading from error commission to subsequent awareness revealed using EEG source imaging. PLoS ONE, 6, e19578. doi:10.1371 /journal.pone.0019578

Dreisbach, G., \& Goschke, T. (2004). How positive affect modulates cognitive control: Reduced perseveration at the cost of increased distractibility. Journal of Experimental Psychology: Learning, Memory, and Cognition, 30, 343-353. doi:10.1037/0278-7393.30.2.343
Endrass, T., \& Ullsperger, M. (2014). Specificity of performance monitoring changes in obsessive-compulsive disorder. Neuroscience \& Biobehavioral Reviews, 46, 124-138. doi:10.1016/j. neubiorev.2014.03.024

Falkenstein, M., Hohnsbein, J., Hoormann, J., \& Blanke, L. (1991). Effects of crossmodal divided attention on late ERP components: II. Error processing in choice reaction tasks. Electroencephalography and Clinical Neurophysiology, 78, 447-455. doi:10.1016/0013-4694(91)90062-9

Falkenstein, M., Hoormann, J., Christ, S., \& Hohnsbein, J. (2000). ERP components on reaction errors and their functional significance: A tutorial. Biological Psychology, 51, 87-107. doi:10.1016/S03010511(99)00031-9

Faul, F., Erdfelder, E., Lang, A. G., \& Buchner, A. (2007). G*Power 3: A flexible statistical power analysis program for the social, behavioral, and biomedical sciences. Behavior Research Methods, 39, 175-191. doi:10.3758/bf03193146

Frank, M. J., D’Lauro, C., \& Curran, T. (2007). Cross-task individual differences in error processing: Neural, electrophysiological, and genetic components. Cognitive, Affective, \& Behavioral Neuroscience, 7, 297-308. doi:10.3758/CABN.7.4.297

Frank, M. J., Woroch, B. S., \& Curran, T. (2005). Error-related negativity predicts reinforcement learning and conflict biases. Neuron, 47, 495-501. doi:10.1016/j.neuron.2005.06.020

Franken, I. H. A., van Strien, J. W., Franzek, E. J., \& van de Wetering, B. J. (2007). Error-processing deficits in patients with cocaine dependence. Biological Psychology, 75, 45-51. doi:10.1016/j. biopsycho.2006.11.003

Fredrickson, B. L. (1998). What good are positive emotions? Review of General Psychology, 2, 300-319. doi:10.1037/1089-2680.2.3.300. What

Fredrickson, B. L. (2001). The role of positive emotions in positive psychology: The broaden-and-build theory of positive emotions. American Psychologist, 56, 218-226. doi:10.1037/0003-066 X.56.3.218

Fredrickson, B. L. (2004). The broaden-and-build theory of positive emotions. Philosophical Transactions of the Royal Society B, 359, $1367-$ 1378. doi:10.1098/rstb.2004.1512

Fuchs, M., Kastner, J., Wagner, M., Hawes, S., \& Ebersole, J. S. (2002). A standardized boundary element method volume conductor model. Clinical Neurophysiology, 113, 702-712. doi:10.1016/S1388-2457 (02)00030-5

Gehring, W. J., Goss, B., Coles, M. G. H. H., Meyer, D. E., \& Donchin, E. (1993). A neural system for error detection and compensation. Psychological Science, 4, 385-390. doi:10.1111/j.1467-9280.1993. tb00586.x

Gilet, A. L. (2008). Procédures d'induction d'humeurs en laboratoire: Une revue critique. Encephale, 34, 233-239. doi:10.1016/j. encep.2006.08.003

Gomez, P., Zimmermann, P. G., Schär, S. G., \& Danuser, B. (2009). Valence lasts longer than arousal: Persistence of induced moods as assessed by psychophysiological measures. Journal of Psychophysiology, 23, 717. doi:10.1027/0269-8803.23.1.7

Gratton, G., Coles, M. G. H., \& Donchin, E. (1983). A new method for off-line removal of ocular artifact. Electroencephalography and Clinical Neurophysiology, 55, 468-484. doi:10.1016/0013-4694 (83)90135-9

Hajcak, G., McDonald, N., \& Simons, R. F. (2003a). Anxiety and errorrelated brain activity. Biological Psychology, 64, 77-90. doi:10.1016/S0301-0511(03)00103-0

Hajcak, G., McDonald, N., \& Simons, R. F. (2003b). To err is autonomic: Error-related brain potentials, ANS activity, and post-error compensatory behavior. Psychophysiology, 40, 895-903. doi:10.1111/14698986.00107

Hajcak, G., McDonald, N., \& Simons, R. F. (2004). Error-related psychophysiology and negative affect. Brain and Cognition, 56, 189197. doi:10.1016/j.bandc.2003.11.001 
Herrmann, M. J., Römmler, J., Ehlis, A. C., Heidrich, A., \& Fallgatter, A. J. (2004). Source localization (LORETA) of the error-relatednegativity (ERN/Ne) and positivity $(\mathrm{Pe})$. Cognitive Brain Research, 20, 294-299. doi:10.1016/j.cogbrainres.2004.02.013

Holmes, E. A., Mathews, A., Dalgleish, T., \& Mackintosh, B. (2006). Positive interpretation training: Effects of mental imagery versus verbal training on positive mood. Behavior Therapy, 37, 237-247. doi:10.1016/j.beth.2006.02.002

Holmes, E. A., Oughtrey, A. E., \& Connor, A. (2008). Looking at or through rose-tinted glasses? Imagery perspective and positive mood. Emotion, 8, 875-879. doi:10.1037/a0013617

Holmes, A. J., \& Pizzagalli, D. A. (2008). Spatiotemporal dynamics of error processing dysfunctions in major depressive disorder. Archives of General Psychiatry, 65, 179-188. doi:10.1001 /archgenpsychiatry.2007.19

Holmes, A. J., \& Pizzagalli, D. A. (2010). Effects of task-relevant incentives on the electrophysiological correlates of error processing in major depressive disorder. Cognitive, Affective, \& Behavioral Neuroscience, 10, 119-128. doi:10.3758/CABN.10.1.119

Holroyd, C. B., \& Coles, M. (2002). The neural basis of human error processing: Reinforcement learning, dopamine, and the error-related negativity. Psychological Review, 109, 679-709. doi:10.1037/0033295X.109.4.679

Huntsinger, J. R. (2012). Does positive affect broaden and negative affect narrow attentional scope? A new answer to an old question. Journal of Experimental Psychology: General, 141, 595-600. doi:10.1037 /a0027709

Inzlicht, M., Bartholow, B. D., \& Hirsh, J. B. (2015). Emotional foundations of cognitive control. Trends in Cognitive Sciences, 19, 126 132. doi:10.1016/j.tics.2015.01.004

Inzlicht, M., McGregor, I., Hirsh, J. B., \& Nash, K. (2009). Neural markers of religious conviction. Psychological Science, 20, 385392. doi:10.1111/j.1467-9280.2009.02305.x

Isen, A. M. (2008). Some ways in which positive affect influences decsion making and problem solving. In M. Lewis, J. M. Haviland-Jones, \& L. Feldman Barrett (Eds.), Handbook of emotions (3rd ed., pp. 548573). New York, NY: Guilford Press.

Jurcak, V., Tsuzuki, D., \& Dan, I. (2007). 10/20, 10/10, and 10/5 systems revisited: Their validity as relative head-surface-based positioning systems. NeuroImage, 34, 1600-1611. doi:10.1016 /j.neuroimage.2006.09.024

Keil, A., Debener, S., Gratton, G., Junghöfer, M., Kappenman, E. S., Luck, S. J., \& Yee, C. M. (2014). Committee report: Publication guidelines and recommendations for studies using electroencephalography and magnetoencephalography. Psychophysiology, 51, 121. doi:10.1111/psyp. 12147

Koban, L., Brass, M., Lynn, M. T., \& Pourtois, G. (2012). Placebo analgesia affects brain correlates of error processing. PLoS ONE, 7, e49784. doi:10.1371/journal.pone.0049784

Koban, L., \& Pourtois, G. (2014). Brain systems underlying the affective and social monitoring of actions: An integrative review. Neuroscience \& Biobehavioral Reviews, 46, 71-84. doi:10.1016/j. neubiorev.2014.02.014

Kross, E., Davidson, M., Weber, J., \& Ochsner, K. (2009). Coping with emotions past: The neural bases of regulating affect associated with negative autobiographical memories. Biological Psychiatry, 65, 361-366. doi:10.1016/j.biopsych.2008.10.019

Laming, D. (1979). Autocorrelation of choice-reaction times. Acta Psychologica, 43, 381-412. doi:10.1016/0001-6918(79)90032-5

Larson, M. J., Good, D. a., \& Fair, J. E. (2010). The relationship between performance monitoring, satisfaction with life, and positive personality traits. Biological Psychology, 83, 222-228. doi:10.1016/j. biopsycho.2010.01.003

Leuthold, H., \& Sommer, W. (1999). ERP correlates of error processing in spatial S-R compatibility tasks. Clinical Neurophysiology, 110, 342-357. doi:10.1016/S1388-2457(98)00058-3
Luu, P., Collins, P., \& Tucker, D. M. (2000). Mood, personality, and self-monitoring: Negative affect and emotionality in relation to frontal lobe mechanisms of error monitoring. Journal of Experimental Psychology. General, 129, 43-60. doi:10.1037 /0096-3445.129.1.43

Lykken, D., \& Venables, P. (1971). Direct measurement of skin conductance: A proposal for standardization. Psychophysiology, 8, 656672. doi:10.1111/j.1469-8986.1971.tb00501.x

Mathewson, K. J., Dywan, J., \& Segalowitz, S. J. (2005). Brain bases of error-related ERPs as influenced by age and task. Biological Psychology, 70, 88-104. doi:10.1016/j.biopsycho.2004.12.005

Mazziotta, J., Toga, A., Evans, A., Fox, P., Lancaster, J., Zilles, K., \& Mazoyer, B. (2001). A probabilistic atlas and reference system for the human brain: International Consortium for Brain Mapping (ICBM). Philosophical Transactions of the Royal Society B, 356, 1293-1322. doi:10.1098/rstb.2001.0915

Michel, C. M., Seeck, M., \& Landis, T. (1999). Spatiotemporal dynamics of human cognition. Physiology, 14, 206-214.

Mitchell, R. L. C., \& Phillips, L. H. (2007). The psychological, neurochemical and functional neuroanatomical mediators of the effects of positive and negative mood on executive functions. Neuropsychologia, 45, 617-629. doi:10.1016/j.neuropsychologia.2006.06.030

Moser, J. S., Hajcak, G., \& Simons, R. F. (2005). The effects of fear on performance monitoring and attentional allocation. Psychophysiology, 42, 261-268. doi:10.1111/j.1469-8986.2005.00290.x

Moser, J. S., Moran, T. P., Schroder, H. S., Donnellan, M. B., \& Yeung, N. (2013). On the relationship between anxiety and error monitoring: a meta-analysis and conceptual framework. Frontiers in Human Neuroscience, 7, 466. doi:10.3389/fnhum.2013.00466

Mulert, C., Jäger, L., Schmitt, R., Bussfeld, P., Pogarell, O., Möller, H. J., \& Hegerl, U. (2004). Integration of fMRI and simultaneous EEG: Towards a comprehensive understanding of localization and timecourse of brain activity in target detection. NeuroImage, 22, 83-94. doi:10.1016/j.neuroimage.2003.10.051

Murray, M. M., Brunet, D., \& Michel, C. M. (2008). Topographic ERP analyses: A step-by-step tutorial review. Brain Topography, 20, 249-264. doi:10.1007/s10548-008-0054-5

Nadler, R. T., Rabi, R., \& Minda, J. P. (2010). Better mood and better performance. Learning rule-described categories is enhanced by positive mood. Psychological Science, 21, 1770-1776. doi:10.1177/0956797610387441

Nieuwenhuis, S., Ridderinkhof, K. R., Blom, J., Band, G. P., \& Kok, A. (2001). Error-related brain potentials are differentially related to awareness of response errors: Evidence from an antisaccade task. Psychophysiology, 38, 752-760. doi:10.1111/1469-8986.3850752

Notebaert, W., Houtman, F., Opstal, F. V., Gevers, W., Fias, W., \& Verguts, T. (2009). Post-error slowing: An orienting account. Cognition, 111, 275-279. doi:10.1016/j.cognition.2009.02.002

O'Connell, R. G., Dockree, P. M., Bellgrove, M. A., Kelly, S. P., Hester, R., Garavan, H., \& Foxe, J. J. (2007). The role of cingulate cortex in the detection of errors with and without awareness: A high-density electrical mapping study. European Journal of Neuroscience, 25, 2571-2579. doi:10.1111/j.1460-9568.2007.05477.x

Olvet, D. M., \& Hajcak, G. (2008). The error-related negativity (ERN) and psychopathology: Toward an endophenotype. Clinical Psychology Review, 28, 1343-1354. doi:10.1016/j.cpr.2008.07.003

Olvet, D. M., \& Hajcak, G. (2009). Reliability of error-related brain activity. Brain Research, 1284, 89-99. doi:10.1016/j. brainres.2009.05.079

Olvet, D. M., \& Hajcak, G. (2012). The error-related negativity relates to sadness following mood induction among individuals with high neuroticism. Social Cognitive and Affective Neuroscience, 7, 289295. doi: $10.1093 / \mathrm{scan} / \mathrm{nsr} 007$

Olvet, D. M., Klein, D. N., \& Hajcak, G. (2010). Depression symptom severity and error-related brain activity. Psychiatry Research, 179, 30-37. doi:10.1016/j.psychres.2010.06.008 
Overbeek, T. J. M., Nieuwenhuis, S., \& Ridderinkhof, K. R. (2005). Dissociable components of error processing: On the functional significance of the Pe vis-à-vis the ERN/Ne. Journal of Psychophysiology, 19, 319-329. doi:10.1027/0269-8803.19.4.319

Pascual-Marqui, R. (2002). Standardized low-resolution brain electromagnetic tomography (sLORETA): Technical details. Methods and Findings in Experimental and Clinical Pharmacology, 24(Suppl. D), 5-12.

Pfabigan, D. M., Pintzinger, N. M., Siedek, D. R., Lamm, C., Derntl, B., \& Sailer, U. (2013). Feelings of helplessness increase ERN amplitudes in healthy individuals. Neuropsychologia, 51, 613-621. doi:10.1016/j.neuropsychologia.2012.12.008

Pizzagalli, D. A. (2014). Depression, stress, and anhedonia: Toward a synthesis and integrated model. Annual Review of Clinical Psychology, 10, 393-423. doi:10.1146/annurev-clinpsy-050212185606

Pizzagalli, D. A., Oakes, T. R., Fox, A. S., Chung, M. K., Larson, C. L., Abercrombie, H. C., \& Davidson, R. J. (2004). Functional but not structural subgenual prefrontal cortex abnormalities in melancholia. Molecular Psychiatry, 9(325), 393-405. doi:10.1038/sj. mp.4001501

Pourtois, G. (2011). Early error detection predicted by reduced preresponse control process: An ERP topographic mapping study. Brain Topography, 23, 403-422. doi:10.1007/s10548-010-0159-5

Pourtois, G., Delplanque, S., Michel, C., \& Vuilleumier, P. (2008). Beyond conventional event-related brain potential (ERP): Exploring the time-course of visual emotion processing using topographic and principal component analyses. Brain Topography, 20, 265-277. doi:10.1007/s10548-008-0053-6

Rabbitt, P. M. (1966). Errors and error correction in choice-response tasks. Journal of Experimental Psychology, 71, 264-272. doi: $10.1037 / \mathrm{h} 0022853$

Ridderinkhof, K. R., Ramautar, J. R., \& Wijnen, J. G. (2009). To PE or not to PE: A P3-like ERP component reflecting the processing of response errors. Psychophysiology, 46, 531-538. doi:10.1111 /j.1469-8986.2009.00790.x

Rigoni, D., Pourtois, G., \& Brass, M. (2015). "Why should I care?" Challenging free will attenuates neural reaction to errors. Social Cognitive and Affective Neuroscience, 10, 262-268. doi:10.1093 /scan/nsu068

Ruchsow, M., Spitzer, M., Grön, G., Grothe, J., \& Kiefer, M. (2005). Error processing and impulsiveness in normals: Evidence from event-related potentials. Cognitive Brain Research, 24, 317-325. doi:10.1016/j.cogbrainres.2005.02.003

Rusting, C. L. (1998). Personality, mood, and cognitive processing of emotional information: Three conceptual frameworks. Psychological Bulletin, 124, 165-196. doi:10.1037/0033-2909.124.2.165

Schettino, A., Loeys, T., Delplanque, S., \& Pourtois, G. (2011). Brain dynamics of upstream perceptual processes leading to visual object recognition: A high density ERP topographic mapping study. NeuroImage, 55, 1227-1241. doi:10.1016/j.neuroimage.2011.01.009

Schettino, A., Loeys, T., \& Pourtois, G. (2013). Multiple synergistic effects of emotion and memory on proactive processes leading to scene recognition. NeuroImage, 81, 81-95. doi:10.1016/j.neuroimage.2013.04.115

Schrijvers, D., De Bruijn, E. R., Maas, Y. J., Vancoillie, P., Hulstijn, W., \& Sabbe, B. G. (2009). Action monitoring and depressive symptom reduction in major depressive disorder. International Journal of Psychophysiology, 71, 218-224. doi:10.1016/j.ijpsycho.2008.09.005

Schroder, H. S., Moran, T. P., Infantolino, Z. P., \& Moser, J. S. (2013). The relationship between depressive symptoms and error monitoring during response switching. Cognitive, Affective, \& Behavioral Neuroscience, 13, 790-802. doi:10.3758/s13415-013-0184-4

Schwarz, N., \& Bless, H. (1991). Happy and mindless, but sad and smart? The impact of affective states on analytic reasoning. In J. P. Forgas (Ed.), Emotion and social judgments (pp. 55-71). Oxford, UK: Pergamon Press.
Seeley, W. W., Menon, V., Schatzberg, A. F., Keller, J., Glover, G. H., Kenna, H., \& Greicius, M. D. (2007). Dissociable intrinsic connectivity networks for salience processing and executive control. Journal of Neuroscience, 27, 2349-2356. doi:10.1523 /JNEUROSCI.5587-06.2007

Sharot, T., Korn, C., \& Dolan, R. (2011). How unrealistic optimism is maintained in the face of reality. Nature Neuroscience, 14, 14751479. doi: $10.1038 / \mathrm{nn} .2949$

Sheldon, K. M., \& King, L. (2001). Why positive psychology is necessary. American Psychologist, 56, 216-7. doi:10.1037/0003-066 X.56.3.216

Shiota, M. N., Neufeld, S. L., Danvers, A. F., Osborne, E. A., Sng, O., \& Yee, C. I. (2014). Positive emotion differentiation: A functional approach. Social and Personality Psychology Compass, 8, 104 117. doi:10.1111/spc3.12092

Shiota, M. N., Neufeld, S. L., Yeung, W. H., Moser, S. E., \& Perea, E. F. (2011). Feeling good: Autonomic nervous system responding in five positive emotions. Emotion, 11, 1368-1378. doi:10.1037/a0024278

Sommer, W., Stürmer, B., Shmuilovich, O., Martin-Loeches, M., \& Schacht, A. (2013). How about lunch? Consequences of the meal context on cognition and emotion. PLoS ONE, 8, e70314. doi:10.1371/journal.pone.0070314

Subramaniam, K., Kounios, J., Parrish, T. B., \& Jung-Beeman, M. (2009). A brain mechanism for facilitation of insight by positive affect. Journal of Cognitive Neuroscience, 21, 415-432. doi:10.1162/jocn.2009.21057

Tamir, M., \& Robinson, M. D. (2007). The happy spotlight: Positive mood and selective attention to rewarding information. Personality and Social Psychology Bulletin, 33, 1124-1136. doi:10.1177 /0146167207301030

Uddin, L. Q. (2014). Salience processing and insular cortical function and dysfunction. Nature Reviews Neuroscience, 16, 55-61. doi:10.1038 /nrn3857

Ullsperger, M., Danielmeier, C., \& Jocham, G. (2014). Neurophysiology of performance monitoring and adaptive behavior. Physiological Reviews, 94, 35-79. doi:10.1152/physrev.00041.2012

Ullsperger, M., Fischer, A. G., Nigbur, R., \& Endrass, T. (2014). Neural mechanisms and temporal dynamics of performance monitoring. Trends in Cognitive Sciences, 18, 259-267. doi:10.1016/j. tics.2014.02.009

Ullsperger, M., Harsay, H. A., Wessel, J. R., \& Ridderinkhof, K. R. (2010). Conscious perception of errors and its relation to the anterior insula. Brain Structure and Function, 214, 629-643. doi:10.1007 /s00429-010-0261-1

Vaidyanathan, U., Nelson, L. D., \& Patrick, C. J. (2012). Clarifying domains of internalizing psychopathology using neurophysiology. Psychological Medicine, 42, 447-459. doi:10.1017 /S0033291711001528

Van Veen, V., \& Carter, C. S. (2002). The timing of action-monitoring processes in the anterior cingulate cortex. Journal of Cognitive Neuroscience, 14, 593-602. doi:10.1162/08989290260045837

van Wouwe, N. C., Band, G. P. H., \& Ridderinkhof, K. R. (2011). Positive affect modulates flexibility and evaluative control. Journal of Cognitive Neuroscience, 23, 524-539. doi:10.1162 jocn.2009.21380

Vanlessen, N., De Raedt, R., Mueller, S. C., Rossi, V., \& Pourtois, G. (2015). Happy and less inhibited? Effects of positive mood on inhibitory control during an antisaccade task revealed using topographic evoked potential mapping. Biological Psychology, 110, 190-200. doi:10.1016/j.biopsycho.2015.07.004

Vanlessen, N., Rossi, V., De Raedt, R., \& Pourtois, G. (2013). Positive emotion broadens attention focus through decreased positionspecific spatial encoding in early visual cortex: Evidence from ERPs. Cognitive, Affective, \& Behavioral Neuroscience, 13, 60 79. doi:10.3758/s13415-012-0130-x 
Vanlessen, N., Rossi, V., De Raedt, R., \& Pourtois, G. (2014). Feeling happy enhances early spatial encoding of peripheral information automatically: Electrophysiological time-course and neural sources. Cognitive, Affective, \& Behavioral Neuroscience, 14, 951-969. doi: 10.3758/s13415-014-0262-2

Vocat, R., Pourtois, G., \& Vuilleumier, P. (2008). Unavoidable errors: A spatio-temporal analysis of time-course and neural sources of evoked potentials associated with error processing in a speeded task. Neuropsychologia, 46, 2545-2555. doi:10.1016/j. neuropsychologia.2008.04.006

Walentowska, W., Moors, A., Paul, K., \& Pourtois, G. (2016). Goal relevance influences performance monitoring at the level of the FRN and P3 components. Psychophysiology, 53, 1020-1033. doi:10.1111/psyp. 12651

Walsh, M., \& Anderson, J. (2012). Learning from experience: eventrelated potential correlates of reward processing, neural adaptation, and behavioral choice. Neuroscience \& Biobehavioral Reviews, 36, 1870-1884. doi:10.1016/j.neubiorev.2012.05.008

Watkins, E. R., \& Moberly, N. J. (2009). Concreteness training reduces dysphoria: A pilot proof-of-principle study. Behaviour Research and Therapy, 47, 48-53. doi:10.1016/j.brat.2008.10.014

Weinberg, A., Kotov, R., \& Proudfit, G. H. (2015). Neural indicators of error processing in generalized anxiety disorder, obsessive- compulsive disorder, and major depressive disorder. Journal of Abnormal Psychology, 124, 172-185. doi:10.1037/abn0000019

Weinberg, A., Riesel, A., \& Hajcak, G. (2012). Integrating multiple perspectives on error-related brain activity: The ERN as a neural indicator of trait defensive reactivity. Motivation and Emotion, 36, 84 100. doi:10.1007/s11031-011-9269-y

Westermann, R., Stahl, G., \& Hesse, F. W. (1996). Relative effectiveness and validity of mood induction procedures: A meta-analysis. European Journal of Social Psychology, 26, 557-580. doi:10.1002 /(SICI)1099-0992(199607)26:4<557::AID-EJSP769>3.0.CO;2-4

Wiswede, D., Münte, T. F., Goschke, T., \& Rüsseler, J. (2009). Modulation of the error-related negativity by induction of shortterm negative affect. Neuropsychologia, 47, 83-90. doi:10.1016/j. neuropsychologia.2008.08.016

Wiswede, D., Münte, T. F., \& Rüsseler, J. (2009). Negative affect induced by derogatory verbal feedback modulates the neural signature of error detection. Social Cognitive and Affective Neuroscience, 4, 227-237. doi:10.1093/scan/nsp015

Zumsteg, D., Friedman, A., Wennberg, R. A., \& Wieser, H. G. (2005). Source localization of mesial temporal interictal epileptiform discharges: Correlation with intracranial foramen ovale electrode recordings. Clinical Neurophysiology, 116, 2810-2818. doi:10.1016 /j.clinph.2005.08.009 